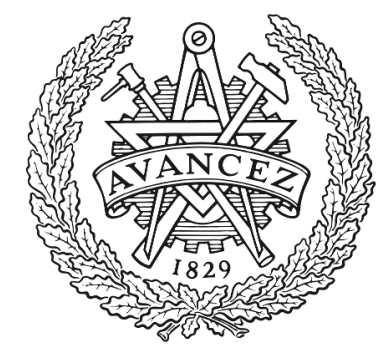

\title{
CHALMERS
}

UNIVERSITY OF TECHNOLOGY

\section{Improved prediction of sheet cavitation inception using bridged transition sensitive turbulence model and cavitation model}

Downloaded from: https://research.chalmers.se, 2023-04-26 13:09 UTC

Citation for the original published paper (version of record):

Ge, M., Svennberg, U., Bensow, R. (2021). Improved prediction of sheet cavitation inception using bridged transition sensitive turbulence model and cavitation model. Journal of Marine Science and Engineering, 9(12). http://dx.doi.org/10.3390/jmse9121343

N.B. When citing this work, cite the original published paper. 
Article

\title{
Improved Prediction of Sheet Cavitation Inception Using Bridged Transition Sensitive Turbulence Model and Cavitation Model
}

\author{
Muye Ge ${ }^{1, *}$, Urban Svennberg ${ }^{2}$ and Rickard E. Bensow ${ }^{1}$ (D) \\ 1 Department of Mechanics and Maritime Sciences, Chalmers Univerisity of Technology, \\ 41296 Gothenburg, Sweden; rickard.bensow@chalmers.se \\ 2 Kongsberg Hydrodynamic Research Centre, 68193 Kristinehamn, Sweden; \\ urban.svennberg@km.kongsberg.com \\ * Correspondence: muye.ge@chalmers.se; Tel.: +46-(0)-704233698
}

check for updates

Citation: Ge, M.; Svennberg, U.; Bensow, R.E. Improved Prediction of Sheet Cavitation Inception Using Bridged Transition Sensitive Turbulence Model and Cavitation Model. J. Mar. Sci. Eng. 2021, 9, 1343. https://doi.org/10.3390/jmse9121343

Academic Editor: Kourosh Koushan

Received: 2 November 2021

Accepted: 22 November 2021

Published: 29 November 2021

Publisher's Note: MDPI stays neutral with regard to jurisdictional claims in published maps and institutional affiliations.

Copyright: (c) 2021 by the authors. Licensee MDPI, Basel, Switzerland. This article is an open access article distributed under the terms and conditions of the Creative Commons Attribution (CC BY) license (https:/ / creativecommons.org/licenses/by/ $4.0 /)$.

\begin{abstract}
Sheet cavitation inception can be influenced by laminar boundary layer flow separation under Reynolds numbers regimes with transitional flow. The lack of accurate prediction of laminar separation may lead to massive over-prediction of sheet cavitation under certain circumstances, including model scale hydrofoils and marine propellers operating at relatively low Reynolds number. For non-cavitating flows, the local correlation based transition model, $\gamma-R e_{\theta}$ transition model, has been found to provide predictions of laminar separation and resulting boundary layer transition. In the present study, the predicted laminar separation using $\gamma-R e_{\theta}$ transition model is bridged with a cavitation mass transfer model to improve sheet cavitation predictions on hydrofoils and model scale marine propellers. The bridged model is developed and applied to study laminar separation and sheet cavitation predictions on the NACA16012 hydrofoil under different Reynolds numbers and angles of attack. As a reference case, the open case of the PPTC VP1304 model scale marine propeller tested on an inclined shaft is studied. Lastly as an application case, the predictions of cavitation on a commercial marine propeller from Kongsberg is presented for model scale conditions. Simulations using the bridged model and the standard unbridged approach with $k-\omega S S T$ turbulence model are performed using the open-source package OpenFOAM, both using the Schnerr-Sauer cavitation mass transfer model, and the respective results are compared with available experimental results. The predictions using the bridged model agree well compared to experimental measurements and show significant improvements compared to the unbridged approach.
\end{abstract}

Keywords: RANS; $\gamma-\operatorname{Re}_{\theta}$ turbulence model; sheet cavitation inception; laminar-turbulent transition; laminar separation

\section{Introduction}

Cavitation is one of the common phenomenon that can be found on hydrofoils and machineries operating in water with rotating blades. When local pressure drops below saturation pressure, free-stream nuclei start to grow and develop to different forms of cavitation, which can be classified as traveling bubble cavitation, attached sheet cavitation and vortex cavitation [1]. Knowledge about the sheet cavitation inception mechanism is still in development. Many experimental studies show that seemingly local boundary layer separation is a pre-requisite for sheet cavitation inception, otherwise traveling bubble cavitation will develop that will not stay attached to the wall surface [2-6]. In the study by Franc and Michel [2], experiments were performed on a NACA16012 foil and an elliptical cylinder, and the major observation is that the cavity detachment point is not the minimum pressure point, but downstream of a laminar separation; cavitation is suppressed by laminar boundary layer upstream the sheet cavitation inception location where pressure is below saturation pressure. In [7], the same hydrofoil NACA16012 was studied, and the 
same observation was reported regarding the relationship between laminar separation and cavitation inception. Here, intense traveling bubbles were observed in regions where the pressure was below saturation pressure upstream of the sheet cavitation inception location; this indicates that the occurrence of traveling bubble cavitation may be related with water quality. There are studies suggesting that the laminar separation plays the role of concentrating free-stream nuclei, and by this process sheet cavitation can develop locally and expand both upstream and downstream. There are, however, also studies suggesting that the pressure fluctuations are very intense inside the laminar separation zone and the pressure fluctuations might play an important role interacting with the nuclei dynamics [8-10]. Besides, there are other studies, e.g., [11], showing that sheet cavitation may start developing without flow separation, still sufficient adverse pressure gradient and local thickening of boundary layer are deemed necessary for sheet cavitation inception [12].

Full scale marine propellers typically have a diameter of several meters and are operating under very high Reynolds numbers with a turbulent boundary layer. However, model scale propellers are usually tested inside medium-size cavitation tunnels with typical blade Reynolds numbers within the range of $5 \times 10^{5}-2 \times 10^{6}$, calculated based on blade chord length at $0.7-0.8$ radius. Then, the laminar and transitional boundary layer can be found on the propeller blades. With a Reynolds number higher than $5 \times 10^{5}$, the influence of this on propeller propulsion characteristics is usually small, and avoiding laminartransitional flow requires a much higher Reynolds number [13]. When depressurizing the cavitation tunnel for propeller cavitation tests, the existence of the laminar and transitional boundary layer may lead to laminar suppression of sheet cavitation. To overcome this issue, leading edge roughness can be applied to trigger turbulence. However, this approach is more common for propellers tested in large depressurized wave basins required to run under operating conditions following Froude's scaling law with relatively lower blade Reynolds number; in many tests in medium-size or large-size cavitation tunnels, there are no special treatments.

This phenomena, sheet cavitation suppression by blade laminar flow, is not rare. An example can be found for the propeller PPTC VP1304 used in the second international workshop on cavitation and propeller performance (SMP'15) [14]: the model scale marine propeller was operating mounted on an inclined shaft under both cavitating and noncavitating operating conditions. For cavitating conditions, rather massive traveling bubble cavitation was found on a large region on the propeller blades with sheet cavitation formed only at outer radii. Many participants reported numerical predictions of propeller performance and cavitation patterns summarized in the workshop report; in certain operating conditions, almost all the participants got massively over-prediction of sheet cavitation on propeller blades, where intense traveling bubble cavitation exists in the experiments.

There are many numerical methods to predict cavitation and related side effects, including cavitation induced pressure pulses, noise, erosion and performance changes. The potential flow theory-based numerical methods are widely used for prediction of propeller propulsion performance and cavitation pattern in very early design stage, but due to its theoretical limitations, boundary layer and flow separation can hardly be predicted and cavitation is kept attached to the blade surface. Viscous CFD codes are becoming more widely used for more accurate predictions, usually with turbulence models based on fully turbulent assumption and with cavitation mass transfer models to account for the prediction of cavitation. However, in most of the cavitation mass transfer models, mass transfer is determined by the sign of local pressure and water saturation pressure $\left(p_{\text {local }}-p_{\text {saturation }}\right)$, which means that cavitation can start developing once the local pressure drop below saturation pressure, regardless of whether the boundary layer is laminar or turbulent, as this has little influence on the pressure. This leads to that in numerical simulations, sheet cavitation can be predicted anywhere where the local pressure is below saturation pressure, including regions with laminar or transitional boundary layers, while in experiments, sheet cavitation may be suppressed and possibly only traveling bubble cavitation can appear. 
In order to improve numerical predictions for the discussed problem, seemingly at least the laminar and transitional boundary layer and laminar separation need to be predicted. As the most widely used approach with a long history, Reynolds-Averaged Navier-Stokes Equations (RANS) turbulence models are limited in accuracy for the prediction of laminar and transitional boundary layers. Theoretically, DNS is the most accurate approach that directly solves the N-S equation, and in [10], the laminar separation bubble on the NACA66 hydrofoil with Reynolds number of 45,000 and angle of attack (AoA) $=4^{\circ}$ was predicted. Well resolved Large Eddy Simulation (LES) can be used to predict laminar separation as well and reported in several studies, including [15], in which LES predicted laminar separation was compared with several different approaches. However, DNS and resolved LES are too computationally expensive for most engineering approaches, especially where the focus is on cases with Reynolds numbers around $1 \times 10^{6}$ and complex geometries. Thus, using RANS with the $\gamma-R e_{\theta}$ transition model, based on Local Correlation-based Transition Modelling (LCTM) [16,17], which links empirical transition correlations and local determined flow quantities, appears to be a suitable engineering choice. The transition sensitive turbulence model seems to be capable in predicting natural transition, bypass transition and separation induced transition in the framework of a RANS turbulence model. Especially, the prediction of separation induced transition is claimed to be a 'major advantage' of the model [18], and the locations of laminar separation are well predicted compared with advanced approaches [15]. The fundamental correlation of the transition model is the linking of scaled ratio of vorticity Reynolds number, $R e_{v}=\frac{\rho y^{2}}{\mu} S$, and momentum thickness Reynolds number, $R e_{\theta}$, with the boundary layer shape factor $H$, as $\frac{\max \left(R e_{y}\right)}{2.193 R e_{\theta}} \sim H$. Separation induced transition is triggered by the parameter $\gamma_{\text {sep }}$, which is calculated similarly as $\frac{\max \left(R e_{y}\right)}{3.235 R e_{\theta}}$, where a boundary layer shape factor $H$ higher than 3.5 is used as the correlated laminar separation criteria. The model has been widely used for various applications including application on model scale marine propellers with predictions of separation locations, laminar-transitional flow regions and propeller propulsion characteristics. Nevertheless, for marine propellers in cavitating condition predictions, directly using the transition sensitive model leads to only minor differences in the predicted cavitation pattern compared to predictions using fully turbulent models, as shown in [19]. The reason is that the cavitation mass transfer model still depends on the sign of $p_{\text {local }}-p_{\text {saturation }}$ as the main mass transfer criterion, and the pressure distribution on the propeller blades is not fundamentally changed using the transition sensitive turbulence model, and there are no interactions between the predicted laminar-transitional flow properties and the mass transfer model.

In the present study, the $\gamma-R e_{\theta}$ transition model and the Schnerr-Sauer mass transfer model [20] are bridged via a parameter SepInd, based on the correlation parameter $\gamma_{s e p}$ in the transition model determined by local flow properties, which provides control of the vaporization rate in the cavitation model, while the transition model and cavitation model are kept intact. The numerical models and modifications are presented in Section 2. The different cases used in the study are described in Section 3. Series of 2D and 3D simulations are presented in Section 4 for the validation of the model on the NACA16012 hydrofoil with different angles of attack. The cavitation pattern predictions agree surprisingly well with experimental observations and are significantly improved compared to unbridged model predictions. In Section 5, the studies on the PPTC VP1304 propeller mounted on an inclined shaft, with operating condition corresponding to the condition 2.1 in the SMP'15 workshop is presented. In Section 6, the application in model scale on a commercial marine propeller design from Kongsberg is presented, including studies of the influence of free stream turbulence intensity levels. The bridged model predict significantly improved cavitation pattern predictions as well for these propeller cases. All the numerical simulations are conducted using the open-source package OpenFOAM-v1912. 


\section{Numerical Models}

\subsection{Cavitation Modeling}

The single fluid homogeneous mixture approach is used to represent the two phases of water and vapor as

$$
\rho_{m}=\alpha_{l} \rho_{l}+\left(1-\alpha_{l}\right) \rho_{v}, \quad \mu_{m}=\alpha_{l} \mu_{l}+\left(1-\alpha_{l}\right) \mu_{v}, \quad \alpha_{l}+\alpha_{v}=1,
$$

in which the subscripts $l$ and $v$ represent liquid phase and vapor phase quantities, respectively, $\rho$ represents density, $\mu$ represents dynamic viscosity and $\alpha$ represents volume fraction of the phase. The transport equation of $\alpha$ with mass transfer source terms can be written as

$$
\frac{\partial \alpha_{l}}{\partial t}+\nabla \cdot \alpha_{l} \mathbf{U}=\frac{\dot{m}}{\rho_{l}}=\left(\frac{1}{\rho_{l}}-\alpha_{l}\left(\frac{1}{\rho_{l}}-\frac{1}{\rho_{v}}\right)\right) \dot{m}+\alpha_{l}\left(\frac{1}{\rho_{l}}-\frac{1}{\rho_{v}}\right) \dot{m} .
$$

The cavitation mass transfer model by Schnerr and Sauer [20] is used. The mass transfer term in Equation (2) is decomposed into two terms as

$$
\dot{m}=\alpha_{l} \dot{m}_{a v}+\left(1-\alpha_{l}\right) \dot{m}_{a c}
$$

while the two terms $\dot{m}_{a v}$ and $\dot{m}_{a c}$ are derived based on uniformly distributed spherical nuclei and simplified Rayleigh relations as

$$
\begin{gathered}
\dot{m}_{a c}=C_{c} \alpha_{l} \frac{3 \rho_{l} \rho_{v}}{\rho_{m} R} \sqrt{\frac{2}{3 \rho_{l}}} \sqrt{\frac{1}{\left|p-p_{\text {threhold }}\right|}} \max \left(p-p_{\text {threhold }}, 0\right), \\
\dot{m}_{a v}=C_{v}\left(1+\alpha_{n u c}-\alpha_{l}\right) \frac{3 \rho_{l} \rho_{v}}{\rho_{m} R} \sqrt{\frac{2}{3 \rho_{l}} \sqrt{\frac{1}{\left|p-p_{\text {threhold }}\right|}}} \min \left(p-p_{\text {threhold }}, 0\right),
\end{gathered}
$$

in which $C_{v}$ and $C_{c}$ represent the vaporization and condensation constants.

\subsection{Governing Equations and Turbulence Modeling}

For the RANS approach, the conservation equations of mass and momentum for the homogeneously mixture fluid are

$$
\begin{gathered}
\frac{\partial \rho_{m}}{\partial t}+\nabla \cdot\left(\rho_{m} \mathbf{U}\right)=0 \\
\frac{\partial \rho_{m} \mathbf{U}}{\partial t}+\nabla \cdot\left(\rho_{m} \mathbf{U U}\right)=\nabla \cdot\left(\boldsymbol{\tau}-\rho_{m} \overline{\mathbf{u}^{\prime} \mathbf{u}^{\prime}}\right)-\nabla p+F_{s}
\end{gathered}
$$

and the Boussinesq hypothesis is used for Reynolds stress tensor $\rho_{m} \overline{\mathbf{u}^{\prime} \mathbf{u}^{\prime}}$ modeling,

$$
-\rho_{m} \overline{\mathbf{u}^{\prime} \mathbf{u}^{\prime}}=\mu_{t}\left(\nabla \mathbf{U}+(\nabla \mathbf{U})^{T}\right)-\frac{2}{3} \rho_{m} k \mathbf{I},
$$

where $\mathbf{U}$ represents time averaged velocity, $\mathbf{u}^{\prime}$ the time-varying fluctuating component of velocity, $\mu_{t}$ the turbulent viscosity, $F_{s}$ body forces, $\rho_{m}$ the mixture density, $\tau$ the averaged stress tensor, and I the identity tensor. The vapor density $\rho_{v}$ and water density $\rho_{l}$ are assumed to be constant, while the mixture density $\rho_{m}$ is calculated based on volume fractions $\alpha_{l}$ and $\alpha_{v}$. Turbulent viscosity is modeled using the $\gamma-R e_{\theta}$ coupled with the $k-\omega S S T$ turbulence model as $\mu_{t}=\rho_{m} k T$, where $T$ represents turbulent time scale and $k$ represents turbulent kinetic energy. The turbulent time scale is calculated as $\min \left(a^{*} / \omega, a_{1} / S F_{2}\right)$, in which $a^{*}, a_{1}$ are model constants, $F_{2}$ is a blending function, $S$ is the absolute value of the strain rate and $\omega$ represents specific dissipation rate. Both $k$ and $\omega$ are modeled via additional Reynolds transport equations. In OpenFOAM, the implementation is based on Menter and Esch [21] with updated coefficients from Menter et al. [22]. 


\subsection{Transition Modeling}

For the $\gamma-R e_{\theta}$ transition model, another two transport equations are used: one for the intermittency $\gamma$ and the other for the transition momentum thickness Reynolds number $\operatorname{Re}_{\theta t}$,

$$
\begin{gathered}
\frac{\partial \rho_{m} \gamma}{\partial t}+\nabla \cdot\left(\rho_{m} \mathbf{U} \gamma\right)=P_{\gamma}-E_{\gamma}+\nabla \cdot\left(\left(\mu_{m}+\frac{\mu_{t}}{\sigma_{f}}\right) \nabla \gamma\right), \\
\frac{\partial \rho_{m} \overline{R e_{\theta t}}}{\partial t}+\nabla \cdot\left(\rho_{m} \mathbf{U} \overline{R e_{\theta t}}\right)=P_{\theta t}+\nabla \cdot\left(\sigma_{\theta t}\left(\mu_{m}+\mu_{t}\right) \nabla \overline{R e_{\theta t}}\right),
\end{gathered}
$$

in which $P_{\gamma}$ and $E_{\gamma}$ represent the production and destruction terms of $\gamma, P_{\theta t}$ is the production term in the $\overline{R e_{\theta t}}$ equation and $\sigma_{f}$ and $\sigma_{\theta t}$ are model constants. For more details of the transition model, we refer to $[17,18]$, and here only the related part used in the present study are discussed.

The fundamental idea of the transition model is to relate the scaled ratio of vorticity Reynolds number $R e_{v}=\frac{\rho y^{2}}{\mu} S$ and the momentum thickness Reynolds number $R e_{\theta}$ to the boundary layer shape factor $H$ as $\frac{\max \left(R e_{v}\right)}{C \cdot R e_{\theta}} \sim H$. The constant $C$ equals to 2.193 for moderate pressure gradients $(2.3<H<2.9)$ and to 3.235 for strong adverse pressure gradients, especially near separation $(H=3.5)$. Most correlations are considered in the $\overline{R e_{\theta t}}$ equation and when the criteria are satisfied and transition starts, source terms in the $\gamma$ equation will be activated and $\gamma$ will increase locally inside the boundary layer. Further, the separation induced transition, $\gamma_{s e p}$ is calculated separately based on $\frac{\max \left(R e_{v}\right)}{C \cdot R e_{\theta c}}-1$ with the constant $C=3.235$ as

$$
\gamma_{\text {sep }}=\min \left(s_{1} \max \left[0,\left(\frac{R e_{v}}{C \cdot R e_{\theta c}}\right)-1\right] F_{\text {reattach }}, 2.0\right) F_{\theta t} .
$$

Here, $s_{1}$ is a model constant, the blending function $F_{\text {reattach }}$ controls the boundary layer reattachment and the blending function $F_{\theta t}$ is equal to 0 in the free stream and 1 in the boundary layer, which is used to limiting the $\gamma_{s e p}$ inside the boundary layer. Finally, the effective intermittency $\gamma_{e f f}$ is calculated as $\gamma_{e f f}=\max \left(\gamma, \gamma_{s e p}\right)$. When coupled with other turbulence models, e.g., $k-\omega S S T$ used in the present study, $\gamma_{e f f}$ controls the production term in the $k$ equation to generate turbulence.

\subsection{Model Bridging Formulation}

To create a transition sensitive cavitation mass transfer model, a first approach could be to modify the vaporization factor $C_{v}$ with the laminar separation criterion as $C_{v} \cdot \gamma_{s e p}$. However, directly using $\gamma_{s e p}$ results in a problem that predicted cavitation can never attach to the wall surfaces leaving viscous sub-layer remaining non-cavitating. This is because, using the transition model, the transition is always triggered at the middle of the boundary layer. This is due to its fundamental formation of the criterion related to the vorticity Reynolds number $R e_{v}=\frac{\rho y^{2}}{\mu} S$, which has its maximum values in the middle of the boundary layer. Thus, the $\gamma_{\text {sep }}$ needs to be modified to cover the region between the predicted location around the middle of boundary layer, and the wall surfaces including the viscous sub-layer. This can be done in many different ways; in the present study it is achieved by using $\gamma_{\text {sep }}$ and the cell valued wall normal direction $\mathbf{n}$, thus using mesh information relying on local properties and suitable for parallelized computations.

To explain the procedure used, note that $\gamma_{s e p}$ is a cell valued scalar with the lower bound of 0 and the upper bound of 2, and when the local value is higher than 0 it activates a source term in the $k$ equation and start to generate turbulence locally turning the laminar boundary into a turbulent one. From this, a parameter SepInd in the range $0-1$ is formed that controls the mass transfer from liquid to vapor. The first step is to set SepInd $d_{\text {org }}=1.0$ for $\gamma_{\text {sep }}>0$ and SepInd $d_{\text {org }}=0$ otherwise, and set SepInd $=$ SepInd $_{\text {org }}$. Further, the limiting function $F_{\theta t}$ in the original LCTM is changed to the explicit criterion 
$F_{\theta t}>0.95$ for non-cavitating regions for robustness. Next step is to perform an ad hoc transportation of SepInd. Here, the transportation is not based on velocity, instead for the present purpose SepInd is transported by the normalized cell wall normal direction defined as $\mathbf{n}$, which is a unit vector pointing from the local cell center to the nearest wall surface. The transportation is solved in an explicit way with special modifications. In order to construct the normalized 'face flux' of SepInd, the face value of $\mathbf{n}_{f}$ is linearly interpolated from $\mathbf{n}$, and the corresponding face valued SepInd $f$ can be set to 1.0 for a cell with SepInd $=1.0$ and SepInd $f_{f}=0$ otherwise. Thus, the 'face flux' $F_{f}=\mathbf{n}_{f} \cdot \mathbf{S}_{f} /\left|\mathbf{S}_{f}\right| \cdot \operatorname{SepInd}_{f}$, is calculated, where $\mathbf{S}_{f}$ is the face area vector and $\left|\mathbf{S}_{f}\right|$ is the face area magnitude. Clearly, $F_{f}$ has a maximum value of 1.0 for the perfectly parallel adjacent cells closer to the wall and a minimum value of -1.0 for the cells further away from the wall surfaces. It can be noted that the transportation is desired on the direction perpendicular to the wall surfaces, and it is necessary to prevent the transportation on the direction parallel to the wall surfaces. A limiter can be introduced, and in the present study $F_{f}$ is limited to 0 for $\left|\mathbf{n}_{f} \cdot \mathbf{S}_{f}\right|<0.2$, in which the scalar 0.2 corresponds to an angle of 78 degrees, between wall normal direction and face normal direction. As the next step, the Gauss theorem can be used to reconstruct new cell values of $\operatorname{SepInd}_{n e w}$, as $\operatorname{SepInd}_{\text {new }}=1$ for $\operatorname{div}\left(F_{f}\right) \cdot V<0$. Lastly, SepInd can be updated to SepInd + SepInd new, and the process is repeated. If we consider the global summation inside the domain gsum (SepInd) represents the number of cells with SepInd =1.0, gsum (SepInd) will monotonically increase till a converged value to gsum $_{\left(S e p I n d_{\text {final }}\right.}$ ). SepInd final $_{\text {in }}$ is used to feed to the cavitation mass transfer model by replacing the vaporization constant $C_{v}$ with $S_{e p I n d}$ final $\cdot C_{v}$. An example of the effect of this transportation can be found in Section 4 .

The model was firstly developed in [23]. In [24], adjusted modification with similar principles is used with improved sheet cavitation predictions and validated with several detailed experiments.

\section{Simulation Design}

\subsection{NACA16012 Hydrofoil}

The NACA16012 hydrofoil is the first studied case in the present paper for model development and validation. The experiments were performed at Grenoble University and experimental results have been published in detail by Franc and Michel [2]. The hydrofoil was tested in a free-surface channel with different angles of attack, cavitation numbers and Reynolds numbers to study the relationship between laminar separation and sheet cavitation inception, which serve as good cases for the present study. Besides, the transition process and predictions using $\gamma-R e_{\theta}$ are highly dependent on the free-stream turbulence levels, and in the experiments the turbulence rates, $T u=\left(\overline{u^{12}}\right)^{0.5} / U$, were measured using a hot-film anemometer.

The hydrofoil was mounted inside the test section with main dimensions of $1.6 \mathrm{~m} \times 0.12 \mathrm{~m} \times 0.4 \mathrm{~m}(\mathrm{~L} \times \mathrm{W} \times \mathrm{H})$ with a submersion depth of $0.2 \mathrm{~m}$. The foil chord length $c$ is $0.1 \mathrm{~m}$ and its maximum thickness at the relative abscissa 0.5 is $1.2 \mathrm{~cm}$. Colored water was injected close to the leading edge to visualize the boundary layer separation. Under different test conditions, the velocity varies from 3-12 m/s, and the turbulence rate $T u$ varies from $0.12-0.16 \%$. For the present numerical study, the simulation is focused on the conditions with AoA of $0^{\circ}$ and $5^{\circ}$ for both cavitating and non-cavitating conditions, as shown in Table 1. 
Table 1. Studied conditions of the NACA 16012 foil.

\begin{tabular}{cccc}
\hline Angle of Attack (AOA) & Reynolds Number & Cavitation Number $\boldsymbol{~}$ & Turbulence Rate Tu \\
\hline $0^{\circ}$ & 300,000 & Non-cavitating & $T u=0.12 \%$ \\
$0^{\circ}$ & $1,000,000$ & $\sigma=0.045$ & $T u=0.15 \%$ \\
$5^{\circ}$ & 300,000 & Non-cavitating & $T u=0.12 \%$ \\
$5^{\circ}$ & $1,000,000$ & $\sigma=0.045$ & $T u=0.15 \%$ \\
\hline
\end{tabular}

The computational mesh is shown in Figure 1 and generated using Pointwise. A circular region close to the foil is meshed with structured grids and connected with an outer region with unstructured grids. The first cell height on the foil was calculated based on $y^{+} \sim 1$ with inlet velocity of $10.04 \mathrm{~m} / \mathrm{s}$, and 60 prism layers with constant growth ratio of 1.05 was generated to fulfill the requirements by the transition model. The $2 \mathrm{D}$ mesh consists of 0.174 million cells in total. For the 3D simulations, the 2D mesh was uniformly extruded by 30 layers in the span-wise direction to a total width of $5 \mathrm{~cm}$ (half compared to the experimental span length), resulting in 4.86 million cells. The top free-surface was substituted with a slip wall condition and the wall effects of the test section are neglected using a slip boundary condition as well.
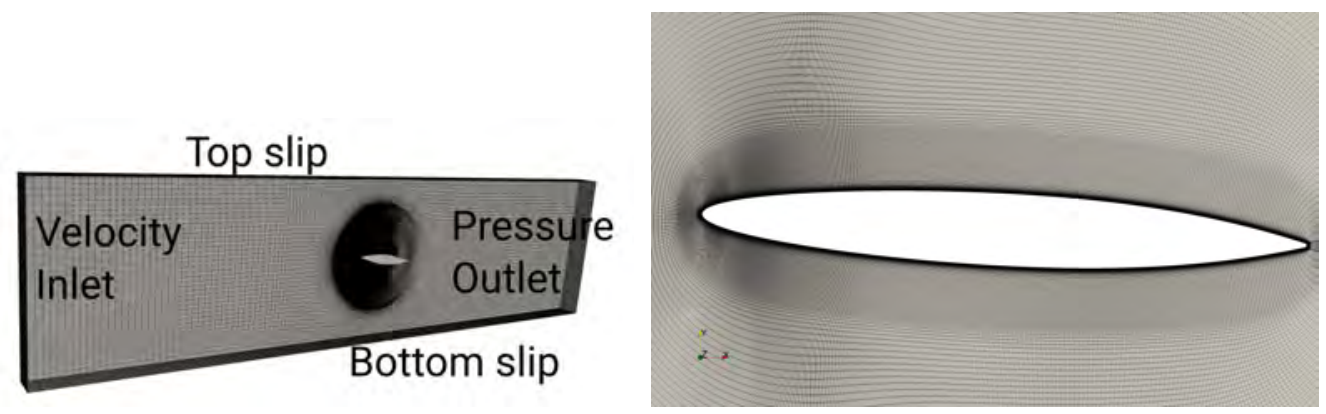

Figure 1. Simulation domain (left) and closer view of computational grids (right).

The water density $\rho_{l}$ and kinematic viscosity $v_{l}$ are chosen to be $998.0 \mathrm{~kg} / \mathrm{m}^{3}$ and $1.004 \times 10^{-6} \mathrm{~m}^{2} / \mathrm{s}$, with $\rho_{v}=0.01208 \mathrm{~kg} / \mathrm{m}^{3}$ and $v_{v}=7.92 \times 10^{-4} \mathrm{~m}^{2} / \mathrm{s}$ for vapor. The inlet velocities $U$ with Reynolds numbers of 300,000 and 1,000,000 can be calculated to be $3.012 \mathrm{~m} / \mathrm{s}$ and $10.04 \mathrm{~m} / \mathrm{s}$, respectively, and fixed at the velocity inlet. The kinetic energy $k$, turbulent dissipation rate $\omega$ and turbulent viscosity $v_{t}$ at the inlet boundary are estimated via,

$$
\mathrm{Tu}=100 \frac{\sqrt{2 k / 3}}{U}, \quad R_{T}=\frac{\rho k}{\mu \omega},
$$

with fixed viscosity ratio $R_{T}=10$. It can be noted that the $R_{T}=10$ is arbitrary and it controls the decay of free-stream $T u$ from the inlet to the simulated body. In the simulations, turbulence decay control is used, thus the choice of $R_{T}$ is not expected to influence the numerical predictions. The used values of $k$ and $\omega$ at the inlet boundary under different conditions are summarized in Table 2.

Table 2. Inlet boundary conditions of the NACA 16012 foil.

\begin{tabular}{cccccc}
\hline Reynolds Number & $\boldsymbol{U}$ & $\boldsymbol{k}$ & $\boldsymbol{\omega}$ & $\boldsymbol{v}_{\boldsymbol{t}}$ & $\boldsymbol{R e}_{\boldsymbol{\theta} \boldsymbol{t}}$ \\
\hline 300,000 & 3.012 & $1.96 \times 10^{-5}$ & 1.952 & $1.004 \times 10^{-5}$ & 1118 \\
$1,000,000$ & 10.04 & 0.00034 & 33.885 & $1.004 \times 10^{-5}$ & 1094.8 \\
\hline
\end{tabular}

\subsection{PPTC VP1304 High-Skew Propeller, Mounted on Inclined Shaft}

The second studied case is the VP1304 propeller, which was studied at the first and second workshops on cavitation and propeller performance, held on the second and fourth International Symposium on Marine Propulsors [14]. 
During the second workshop, the propeller was mounted on an inclined shaft with an inclination angle of $12^{\circ}$. The propulsion characteristics, cavitation behavior and pressure pulses of the propeller operating in oblique flow were measured and studied by various research groups using various numerical methods, including BEM, RANS and ILES with different cavitation mass transfer models. In the present study, focus is put on the task 2.1 in the original workshop, which is a cavitating case with advance ratio $J=1.019$ and cavitation number $\sigma=2.024$. This can be considered a representative configuration for marine propeller cavitation experiments, i.e., with the propeller mounted on inclined shaft to create propeller inflow variations and thus cavitation dynamics. In the original workshop, the majority of participants reported similar cavitation patterns with massive over-prediction on certain blade locations compared with experimental recordings, with different approaches, mesh resolutions and mesh types, flow solvers, turbulence models and cavitation mass transfer models. Thus, the case can be an interesting validation case.

The main particulars of VP1304 are summarized in Table 3, with the numerical configuration shown in Figure 2.

Table 3. Main characteristics of propeller VP1304.

\begin{tabular}{cccccc}
\hline Diameter & Pitch & Chord Length (0.7 R) & Pitch Ratio (0.7 R) & Skew & No. of Blades \\
\hline $250 \mathrm{~mm}$ & $408.75 \mathrm{~mm}$ & $104.167 \mathrm{~mm}$ & 1.635 & $18.8^{\circ}$ & 5 \\
\hline
\end{tabular}

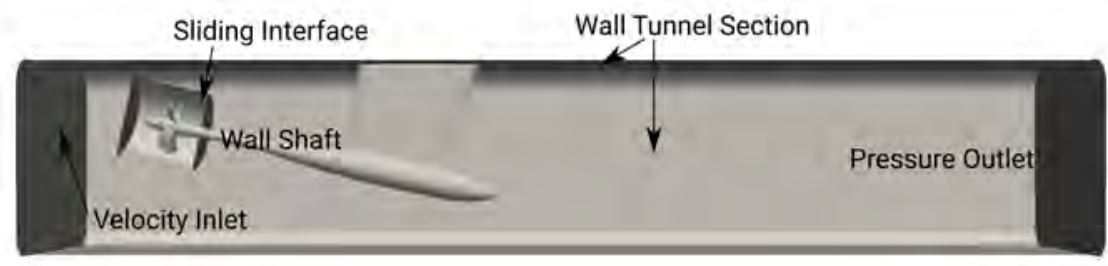

Figure 2. Simulation domain and boundary conditions.

The computational mesh was generated using Pointwise. The blade surfaces were meshed using structured surface mesh, with a hyperbolic extrusion method for generating the conformal prism layer grids. The first layer cell height is $1.5 \times 10^{-6} \mathrm{~m}$ with target $y^{+}=1$, and 44 layers of prism cells were generated, growing from the blade surface with constant growth ratio of 1.15 , and on top of that, another 13 layer prism cells with constant height were generated, resulting in 57 layers of prism cells in total with the height of about $14 \mathrm{~mm}$. Tetrahedral cells were used to fill the rest of the simulation space. A general view of surface and volume mesh is shown in Figure 3 including a close-up view close to the leading edge at the blade tip. There are 27 million cells in the propeller rotation region and 2 million cells for the test section. The cell counts are summarized in Table 4.
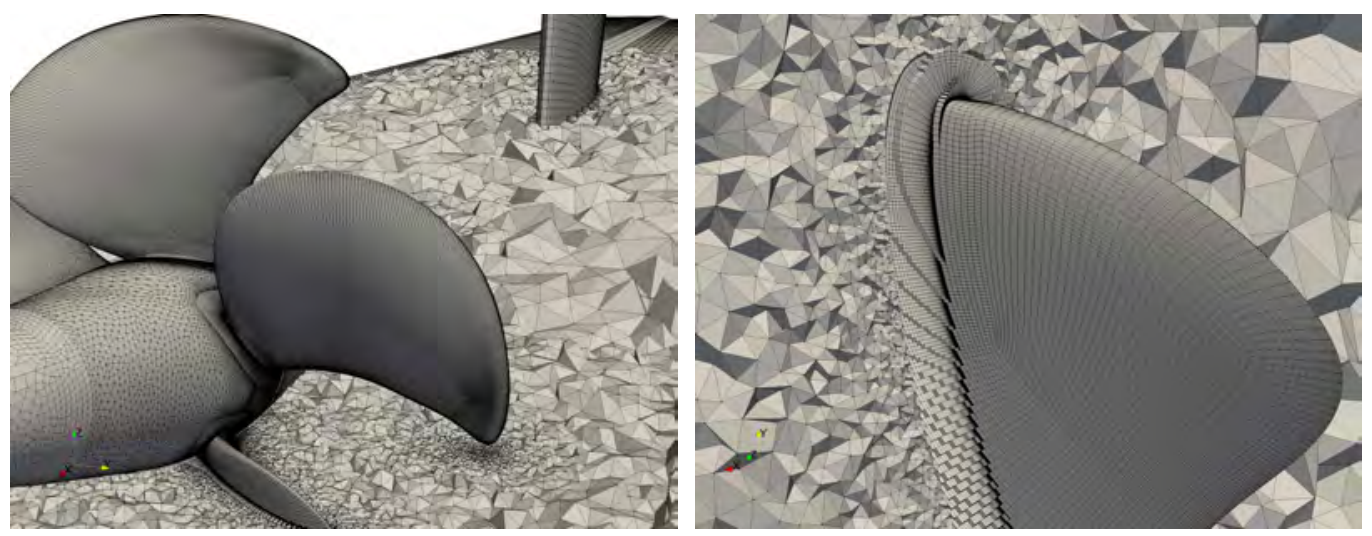

Figure 3. Close-up view at the blade surface mesh and tip volume grids. 
Table 4. Cell counts for case PPTC VP1304.

\begin{tabular}{cccccc}
\hline Region & Tets & Pyramids & Prisms & Hexes & Total \\
\hline Inner & $11.2 \mathrm{Mio}$ & $0.3 \mathrm{Mio}$ & $0.06 \mathrm{Mio}$ & $15.7 \mathrm{Mio}$ & $27.3 \mathrm{Mio}$ \\
Outer & $1.8 \mathrm{Mio}$ & $0.06 \mathrm{Mio}$ & $0.01 \mathrm{Mio}$ & $0.5 \mathrm{Mio}$ & $2.4 \mathrm{Mio}$ \\
Total & $13.0 \mathrm{Mio}$ & $0.36 \mathrm{Mio}$ & $0.31 \mathrm{Mio}$ & $16.2 \mathrm{Mio}$ & $29.7 \mathrm{Mio}$ \\
\hline
\end{tabular}

The simulation conditions are summarized in Table 5, which is the same as the studied condition case 2.1 in the original workshop.

Table 5. Studied operating condition of VP1304.

\begin{tabular}{ccc}
\hline Advance Ratio $J$ & Cavitation Number $\sigma$ & Rotation Speed \\
\hline 1.019 & 2.024 & $1200 \mathrm{rpm}$ \\
\hline Water Density $\rho_{l}$ & Water Kinematic Viscosity $\nu_{l}$ & Saturation Pressure \\
\hline $997.78 \mathrm{~kg} / \mathrm{m}^{3}$ & $9.567 \times 10^{-7} \mathrm{~m}^{2} / \mathrm{s}$ & $2643 \mathrm{~Pa}$ \\
\hline
\end{tabular}

\subsection{Kongsberg High-Skew Propeller Mounted on Inclined Shaft}

As an application example, the last presented case is a commercial high-skew propelled design from Kongsberg tested in model scale, denoted here as propeller A. It was tested in a cavitation tunnel at Kongsberg Hydrodynamic Research Centre. The propeller was mounted on an inclined shaft with about 10 degrees inclination. The cavitation pattern was recorded using high speed video for a propeller operating under different advance ratios $J$ and cavitation numbers $\sigma$. The Reynolds numbers calculated based on blade chord length at $0.75 \mathrm{R}$ ranged between $1,000,000$ to $1,200,000$. In the present study, simulations were performed for an operating condition with $J=0.85$ and $\sigma=2.0$. The computational mesh was generated using Pointwise with two regions: one is the inner propeller rotation region, and one is the outer tunnel region. Mesh refinements were applied at each blade tip vortex region. The target $y^{+}$was 1 and 36 layers of prism cells were extruded from the structured blade surface mesh using hyperbolic extrusion method. The first 15 prism layers had the constant growth ratio of 1.1 to generate suitable boundary layer cells required by the transition model. Tetrahedral cells were used to fill out the rest space. The simulation configuration can be found in Figure 4 with surface and volume grid shown in Figure 5. The summary of the computational grids can be found in Table 6 .

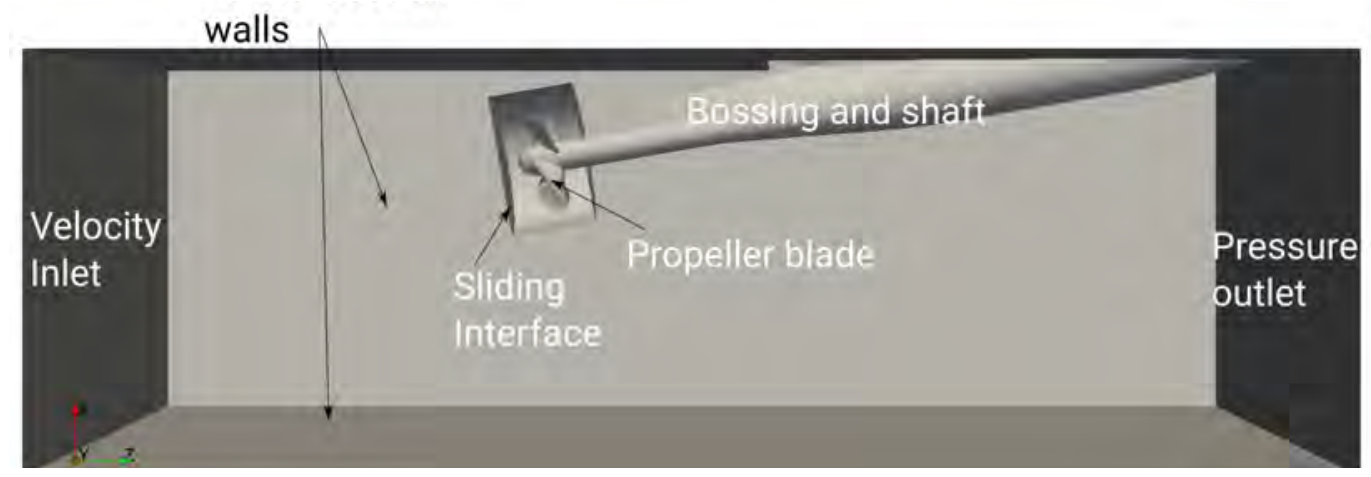

Figure 4. Simulation configuration for propeller A tested in the cavitation tunnel. 


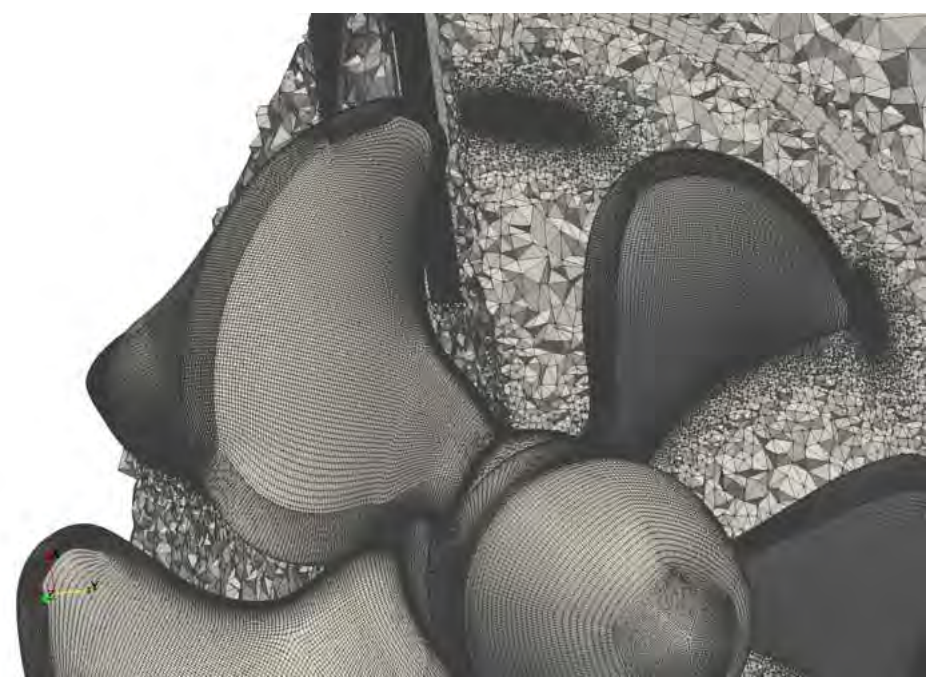

Figure 5. Closer view of surface and volume mesh close to the propeller blades.

Table 6. Cell counts for case Kongsberg propeller A.

\begin{tabular}{cccccc}
\hline Region & Tets & Pyramids & Prisms & Hexes & Total \\
\hline Inner & $11.7 \mathrm{Mio}$ & $0.4 \mathrm{Mio}$ & $0.2 \mathrm{Mio}$ & $10.1 \mathrm{Mio}$ & $22.4 \mathrm{Mio}$ \\
Outer & $2.1 \mathrm{Mio}$ & $0.1 \mathrm{Mio}$ & $0.1 \mathrm{Mio}$ & $1.6 \mathrm{Mio}$ & $3.9 \mathrm{Mio}$ \\
Total & $13.8 \mathrm{Mio}$ & $0.5 \mathrm{Mio}$ & $0.3 \mathrm{Mio}$ & $11.7 \mathrm{Mio}$ & $26.3 \mathrm{Mio}$ \\
\hline
\end{tabular}

\subsection{Numerical Settings}

The Schnerr-Sauer cavitation mass transfer model is used for all the conducted simulations. The cavitation model requires inputs of assumed uniform distributed water nuclei, including the total number per unit water volume $n_{n u c l e i}$ and their diameters $d_{n u c l e i}$, which are fixed to be $10^{12}$ and $1 \times 10^{-6}$ in all of the presented simulations. The vaporization and condensation constants $C_{v}$ and $C_{c}$ are set to 1 .

The second order Backward scheme is used accounting for time advancing; for the convection of velocity and turbulence properties, including $k, \omega, \gamma, R e_{\theta}$, the linearUpwind scheme is used, which blends between the linear interpolation and upwind interpolation based on gradient calculations, for the propeller simulations, while the less diffusive TVD (Total Variation Diminishing) scheme limitedLinear is used for the hydrofoil simulations. The convection of $\alpha$ is discretized using first-order upwind scheme, as the Schnerr-Sauer mass transfer model assumes uniformly distributed nuclei and a simplified RayleighPlesset equation and it is recommended in the original publication [20] for the model. For gradient calculations, the Gauss theorem with linear interpolation is used. For pressurevelocity coupling, the PIMPLE-C algorithm is used for non-cavitating conditions and PIMPLE algorithm is used for cavitating conditions. For the NACA16012 hydrofoil, the SepInd is predicted in the way discussed in Section 2, but for the two marine propellers additional modification is needed, mainly due to mesh reason. For the two marine propellers, the blade prism layers are meshed using structured grid, but pyramids and tetrahedron are used in the outer space. The grid transformation from hexahedron to pyramids or tetrahedron may lead to spurious local strain rate $S$ prediction, which can lead to spurious prediction of $\operatorname{Re}_{v}=\frac{\rho}{v}\left(y^{2} S\right)$. Thus, the SepInd org was limited for non-cavitating regions within blade wall distance within $2 \mathrm{~mm}$ for the PPTC VP1304 case and within $1 \mathrm{~mm}$ for the Kongsberg propeller A for regions with $v_{t}>0.5 v_{l}$. 


\section{NACA 16012 Hydrofoil}

\subsection{Non-Cavitating Condition}

\subsection{1. $\operatorname{Re}=300,000$}

In the experiments, boundary layer separation was visualized by a thin thread of water colored with fluorescein injected close to the foil leading edge. The location of laminar separation is very clearly visible and experimental photographs are reported in the original publication by Franc and Michel [2] for non-cavitating flow with Reynolds number of 300,000 with varying AoA including $0^{\circ}, 3^{\circ}, 4^{\circ}$, and $5^{\circ}$.

Two-dimensional simulations are performed to check the accuracy for the prediction of separation location. The locations of numerically predicted separation are reported based on the cell center location where the $\gamma_{\text {sep }}>0$ is satisfied, which is also the same location where SepInd $=1$ is satisfied. The measured and predicted location of laminar separation is summarized in Table 7, in which the experimental locations are reported based on the photographs in the original paper [2].

At $3^{\circ}$ AoA, the laminar separation can be predicted on the suction side of the foil close to the rear part, located at about $70 \sim 80 \%$ chord length. This position is quite accurately predicted by the $\gamma-R e_{\theta}$ transition model. Besides, the laminar separation is also predicted at the rear part on the foil pressure side. With increasing AoA, the location of laminar separation moves upstream. At $5^{\circ}$, flow circulation at the laminar separation is clearly visible, and its location is at about $3 \sim 5 \%$ chord length. This location is also predicted accurately using the transition model. The foremost location of SepInd can be found at $3.2 \%$ chord length and flow instabilities can be found generated locally and transported downstream inside the boundary layer. Some prediction discrepancies can be found at $4^{\circ}$, but it can be noted that the transition location is very sensitive between AoA of $3^{\circ}$ and $5^{\circ}$, where laminar separation occurs on foil mid chord. A detailed view can be found in Figure 6, including the flow circulation near the leading edge and the predicted SepInd marked in black.

Table 7. Predicted laminar separation location and comparison to experiments, $\operatorname{Re}=300,000$.

\begin{tabular}{ccc}
\hline AoA & Laminar Separation Location (EXP) & Laminar Separation Location (CFD) \\
\hline $0^{\circ}$ & $74 \sim 80 \% c$ & $80 \% c$ \\
$3^{\circ}$ & $73 \sim 77 \% c$ & $74 \% c$ \\
$4^{\circ}$ & $40 \sim 60 \% c$ & $67 \% c$ \\
$5^{\circ}$ & $3 \sim 5 \% c$ & $3 \sim 6 \% c$ \\
\hline
\end{tabular}

The predicted pressure coefficients $(C p)$ along the foil surfaces using $k-\omega S S T$ turbulence model and $\gamma-R e_{\theta}$ transition model with AoA of $0^{\circ}$ and $5^{\circ}$ are shown in Figure 7. The predicted existence of a large region of laminar-transitional boundary layer changes the pressure distribution on the foil compared to the prediction by the fully turbulent turbulence model. This is especially clear at $5-10 \% c$ at $5^{\circ}$ AoA, where local flow circulation is appearing. The separation also induces a series of weak pressure fluctuations downstream, which are predicted, but since the simulation is made in the RANS framework with a correlation based transition model, these fluctuations will not be discussed. 

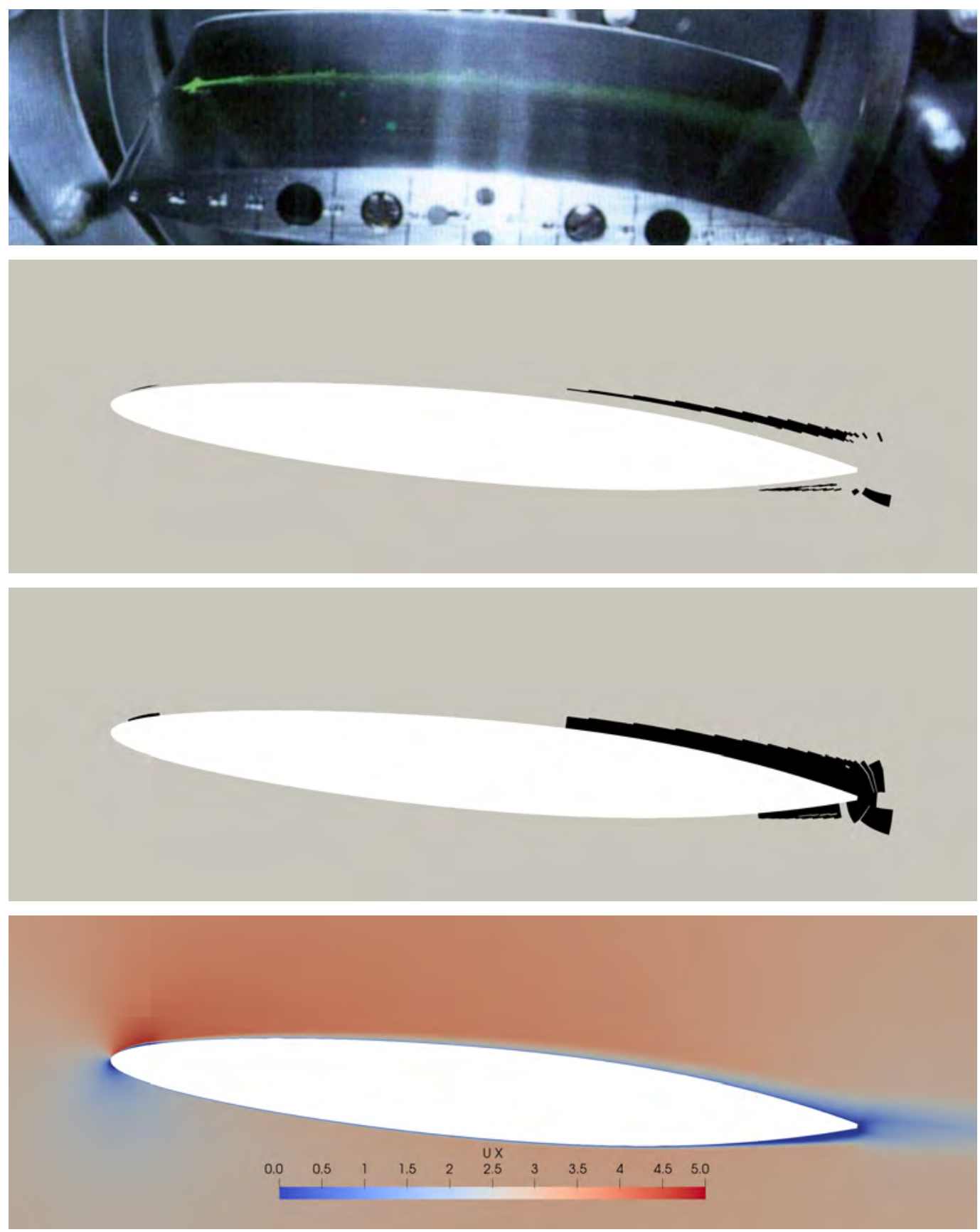

Figure 6. Visualization of laminar transition for NACA16012, $\operatorname{AoA}=5^{\circ}, \operatorname{Re}=300,000$. From top to bottom: experimental photograph; predicted SepInd org $_{\text {; }}$ predicted SepInd; predicted velocity in flow direction.

\subsection{2. $\operatorname{Re}=1,000,000$}

With an increased Reynolds number to $1,000,000$, the predicted $C p$ is shown in Figure 8 and the predicted SepInd and velocity on flow direction is shown in Figure 9. Compared to the predictions with $R e=300,000$, the location of laminar separation is not much influenced, but the boundary layer thickness is comparatively thinner. The separation induced pressure fluctuations also become relatively stronger with a series of local thickening of the boundary layer, as can be seen in Figure 9 based on 2D simulations and Figure 10 based on 3D simulations. 


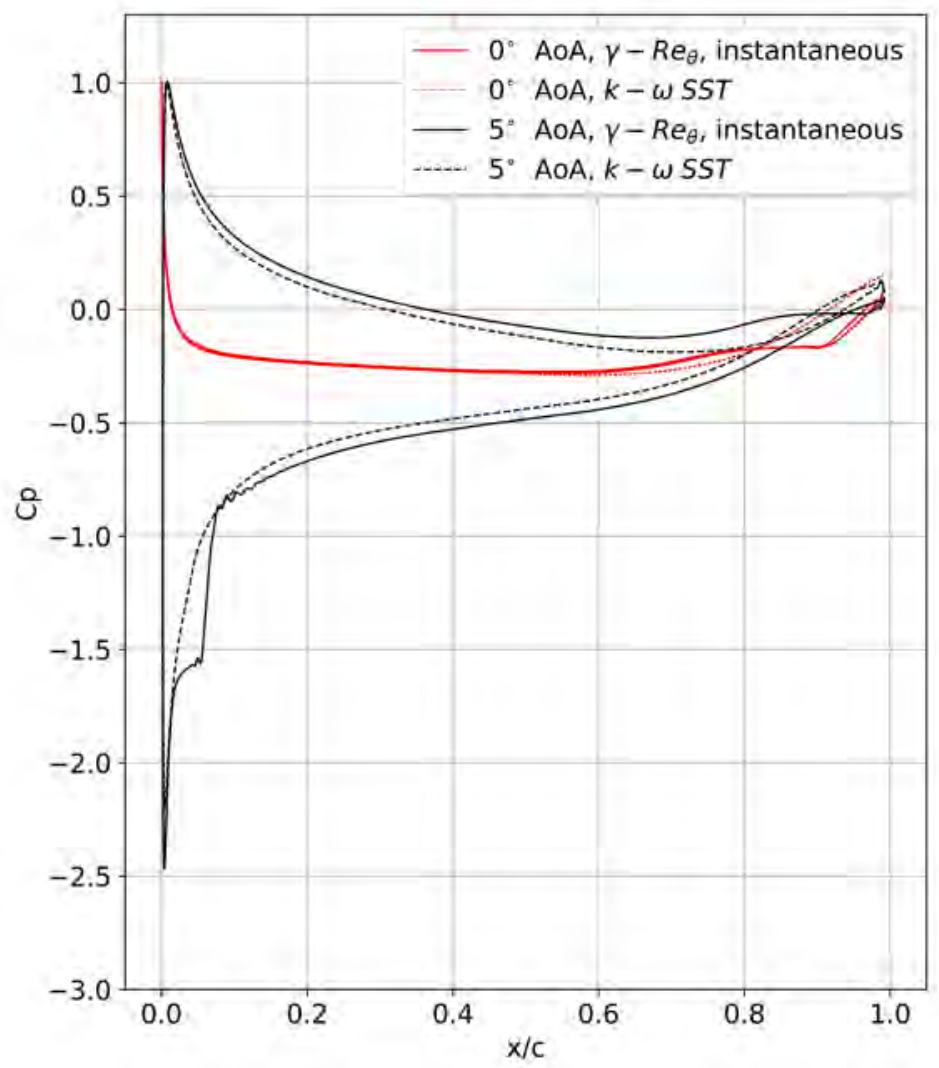

Figure 7. Predicted pressure coefficients at $\mathrm{AoA}$ of $0^{\circ}$ and $5^{\circ}, \operatorname{Re}=300,000$.

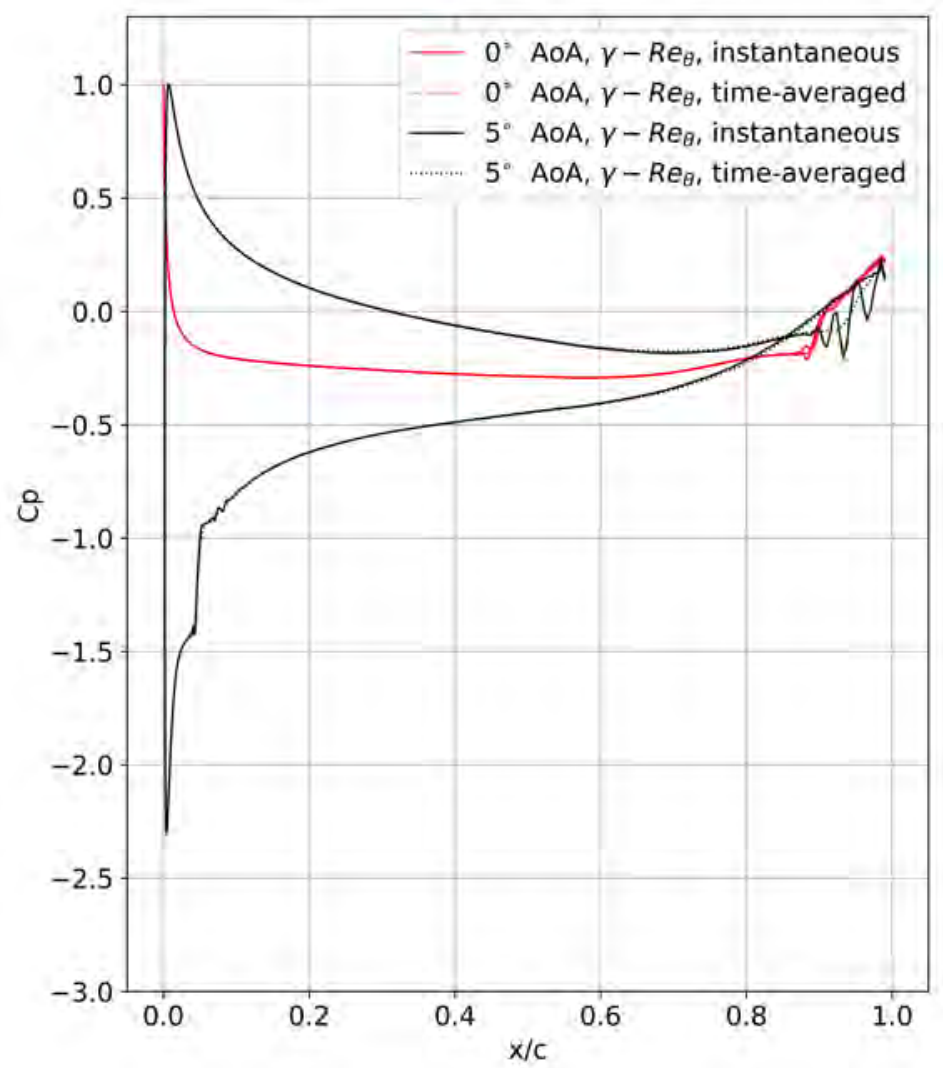

Figure 8. Predicted pressure coefficients at AoA of $0^{\circ}$ and $5^{\circ}, \operatorname{Re}=1,000,000$. 

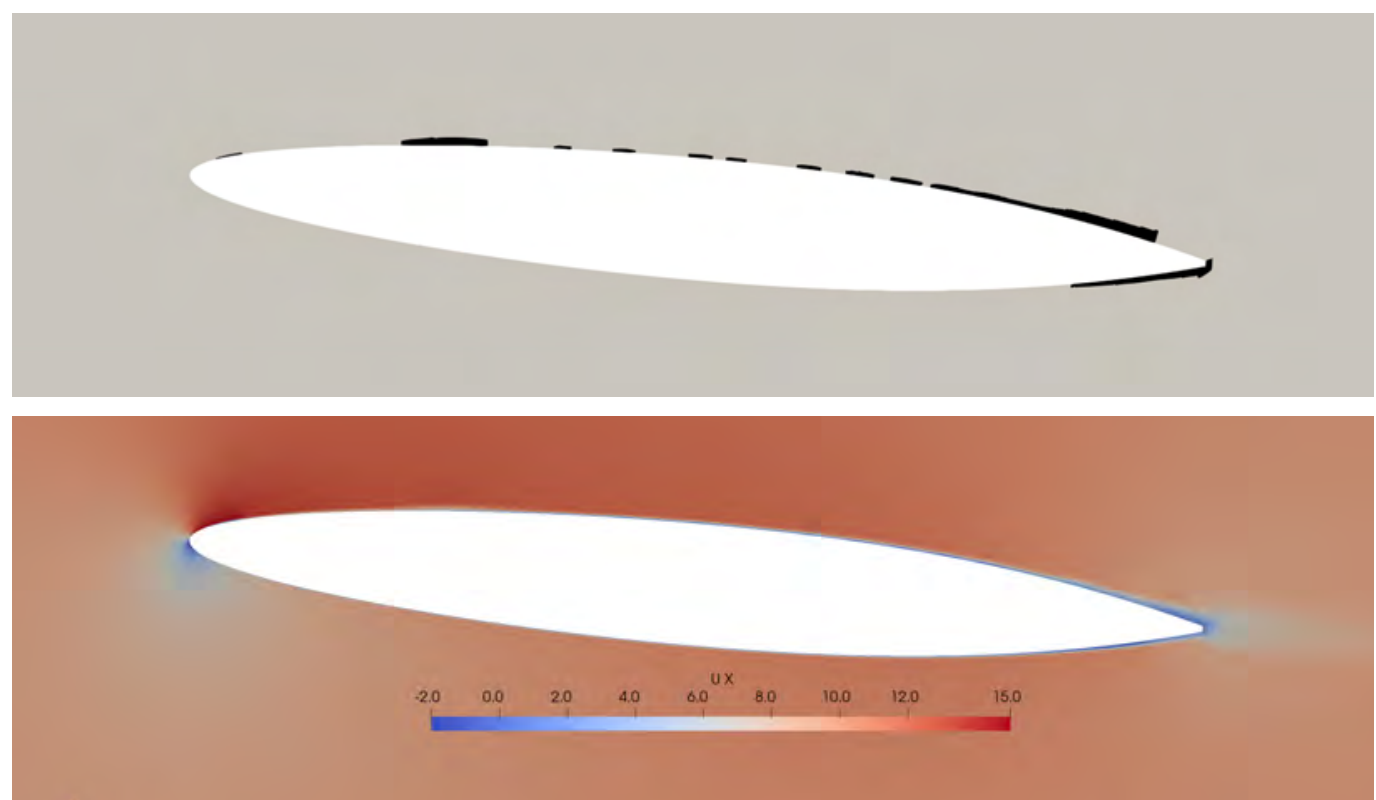

Figure 9. Predicted non-cavitating SepInd (marked in black) and streamwise velocity at Reynolds number of $1,000,000,2 \mathrm{D}$ simulation.

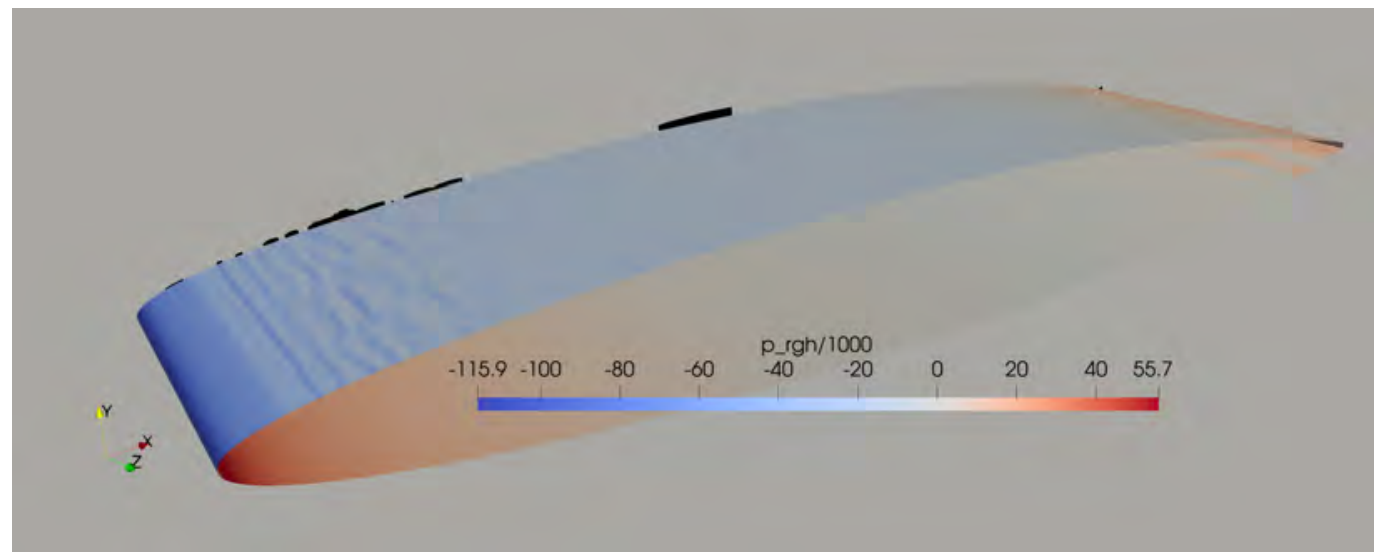

Figure 10. Predicted non-cavitating SepInd (marked in black) and pressure on foil surface at Reynolds number of $1,000,000,3 \mathrm{D}$ simulation.

\subsection{Cavitating Conditions}

The experimental cavitation patterns are shown in Figure 11 for the two angles of attack $0^{\circ}$ and $5^{\circ}$. The numerical predictions using the bridged model, the standard $k-\omega S S T$ turbulence model without transition model and with the unbridged $\gamma-R e_{\theta}$ transition model, together with the Schnerr-Sauer mass transfer model (SS), displaying both 2D and 3D simulations below the experimental photographs.

At $0^{\circ}$ AoA, using the standard $k-\omega S S T$ turbulence model with the Schnerr-Sauer mass transfer cavitation model, rather massive over-prediction of sheet cavitation is predicted with cavitation inception located about $11 \%$ chord length, while in the experiments this inception location is at about $60 \%$ chord length. Almost the same predictions are achieved incorporating the unbridged $\gamma-R e_{\theta}+S S$ transition model, since the pressure field prediction is not much changed and the mass transfer is determined by local pressure. The bridged model predicts cavitation patterns that agree very well compared with the observations of the experiments, where cavitation inception stars at $61 \%$ chord length based on the 2D simulation and $59 \%$ based on the 3D simulation. 

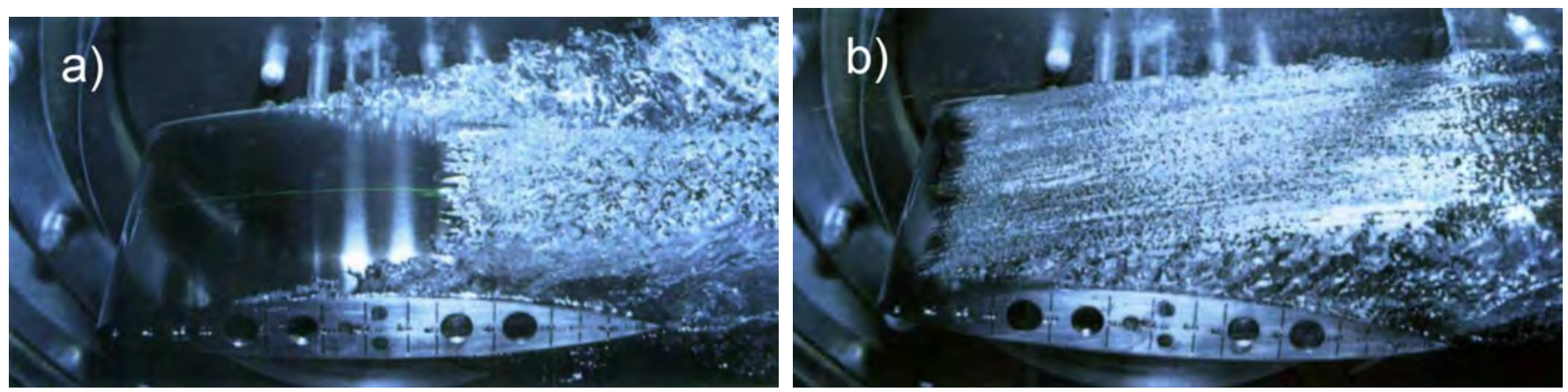

c)

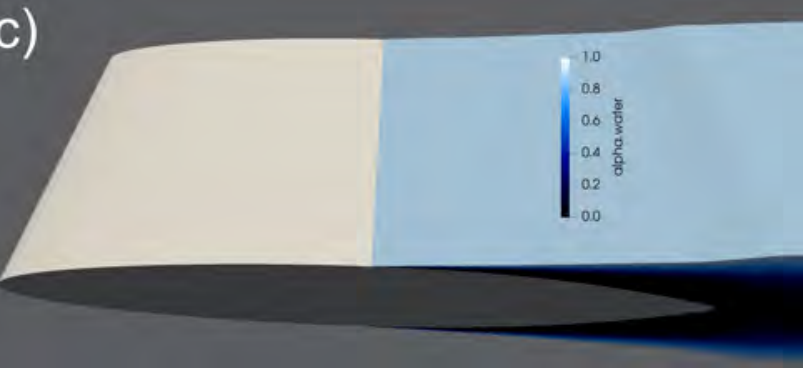

e)

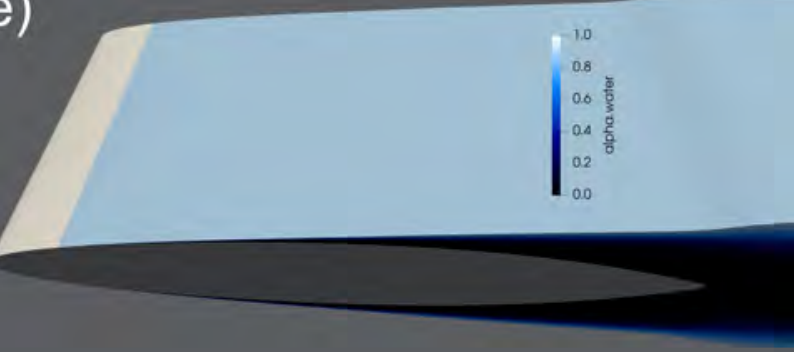

g)
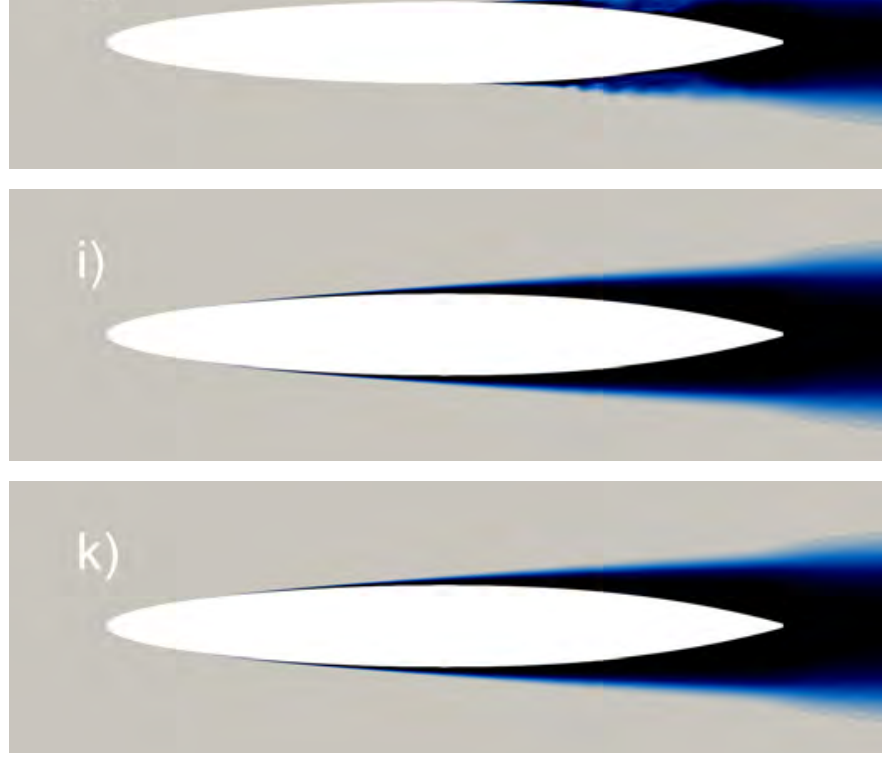
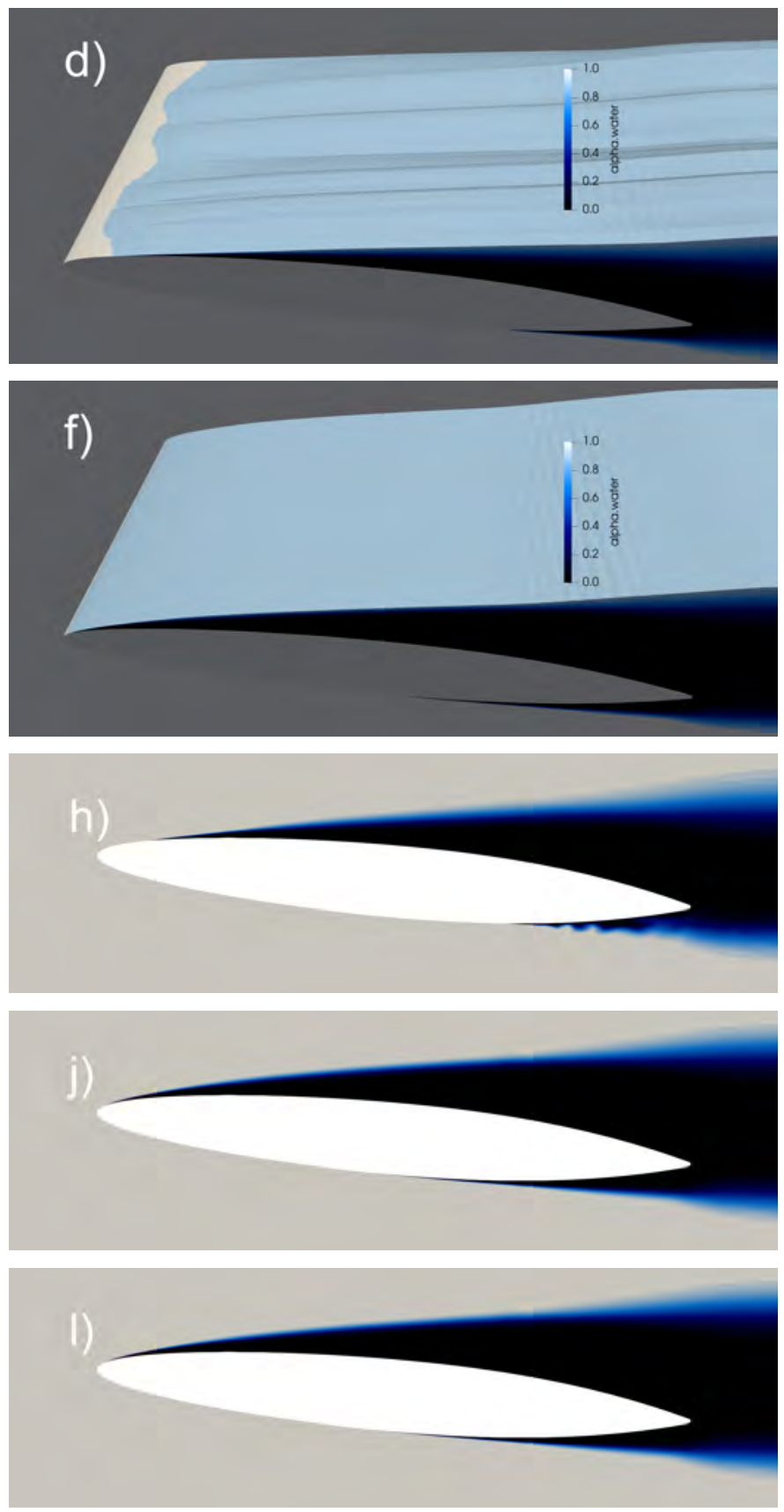

Figure 11. Cavitation patterns on NACA 16012 at AoA of $3^{\circ}$ (left column) and $5^{\circ}$ (right column) with $R e=1,000,000$; $\sigma=0.045$. From top to bottom: (a,b) experimental photos; (c,d) bridged model, 3D; $(\mathbf{e}, \mathbf{f}) k-\omega S S T+S S, 3 \mathrm{D} ;(\mathbf{g}, \mathbf{h})$ bridged model, 2D; (i,j) $k-\omega S S T+S S, 2 \mathrm{D} ;(\mathbf{k}, \mathbf{l}) \gamma-R e_{\theta}+S S, 2 \mathrm{D}$. 
At $5^{\circ}$ AoA, the sheet cavitation starts in the experiments at around $6 \sim 7 \%$ chord length with a clearly span-wise wavy inception line. At this angle of attack, low pressure regions are created starting from the very leading edge and using the $k-\omega S S T$ turbulence model with or without the $\gamma-R e_{\theta}$ transition model the predicted cavity starts from the foil leading edge and covers the whole suction side. The bridged model, on the other hand, predicts the inception location very close to the experimental observation, which is $8.7 \%$ chord length based on the 2D simulation and 5 8\% chord length based on the 3D simulation with a wavy inception line. It can be noted that this wavy inception line predicted in 3D simulation is rather stable and can be related with the flow structures inside the boundary layer close to the leading edge.

Cavitation prediction differences can be found not only on the suction side, but also on the pressure side close to the foil rear part, but the cavitation inception on the pressure side was not studied and reported in detail in the original experiments. A summary of sheet cavitation inception locations on the foil suction side can be found in Table 8 , in which predictions using $2 \mathrm{D}$ simulations for $3^{\circ}$ and $4^{\circ}$ AoA are also reported.

Table 8. Summary of sheet cavitation inception locations.

\begin{tabular}{cccc}
\hline AoA & Exp & Bridged Model & $k-\omega S S T+S S$ \\
\hline $0^{\circ}(2 \mathrm{D})$ & $60 \% c$ & $61 \% c$ & $11 \% c$ \\
$0^{\circ}(3 \mathrm{D})$ & $60 \% c$ & $59 \% c$ & $11 \% c$ \\
$3^{\circ}(2 \mathrm{D})$ & $29-45 \% c$ & $32 \% c$ & $0.1 \% c$ \\
$4^{\circ}(2 \mathrm{D})$ & $13-23 \% c$ & $20.5 \% c$ & $0.1 \% c$ \\
$5^{\circ}(2 \mathrm{D})$ & $6-7 \% c$ & $8.7 \% c$ & $0.1 \% c$ \\
$5^{\circ}(3 \mathrm{D})$ & $6-7 \% c$ & $5-8 \% c$ & $0.1 \% c$ \\
\hline
\end{tabular}

The sheet cavitation development history at $3^{\circ}$ AoA is shown in Figure 12. Cavitating simulations start based on well-developed non-cavitating simulations. At $T=0.001 \mathrm{~s}$, the sheet cavitation starts developing at the same position as the predicted laminar separation close to the rear part. After that, the sheet cavitation front line can be found moving upstream steadily with wavy cavitation interface, and finally the sheet cavitation front line stopped at about $30 \% c$ at about $T=0.05 \mathrm{~s}$.

With $3^{\circ}$ AoA in the experiments, cavitation inception line varies from about $29 \% \mathrm{C}$ from foil center to $45 \% c$ at the foil ends, possibly influenced by test section side walls. The bridged model predicted cavitation inception starts at $32 \% \mathrm{c}$. At $4^{\circ}$ AoA as shown in Figure 13, the bridged model predicted inception starts at $20 \% c$ while in the experiments the inception line varies between $13 \sim 23 \%$ chord length. For these conditions, the unbridged model predict cavitation inception starts at the very leading edge of about $0.1 \% c$. For detailed comparison with experimental photographs, we refer to the original publication [2].

The predicted pressure distributions are plotted in Figure 14. The predictions of pressure distributions $C p$ along the foil surfaces using $k-\omega S S T$ turbulence model with and without the unbridged $\gamma-R e_{\theta}$ transition model are almost identical, and the minimum value is exactly -0.045 under the cavitation number $\sigma=0.045$. The bridged model, which predicted much improved cavitation patterns, predicts large regions where pressure is below saturation pressure. For these regions below saturation pressure, sheet cavitation can not be formed and traveling bubble cavitation may appear, according to the experiments performed on the same geometry in [7] with traveling bubble cavitation. There it was concluded that the NACA16012 hydrofoil with AoA of $3^{\circ}$ clearly displays a relationship between laminar separation and sheet cavitation inception. There are no available experimental data regarding the lift or drag forces of the cavitating foil under these conditions, but from the pressure coefficients shown here in Figure 14, the unbridged approaches may lead to inaccurate force predictions. 

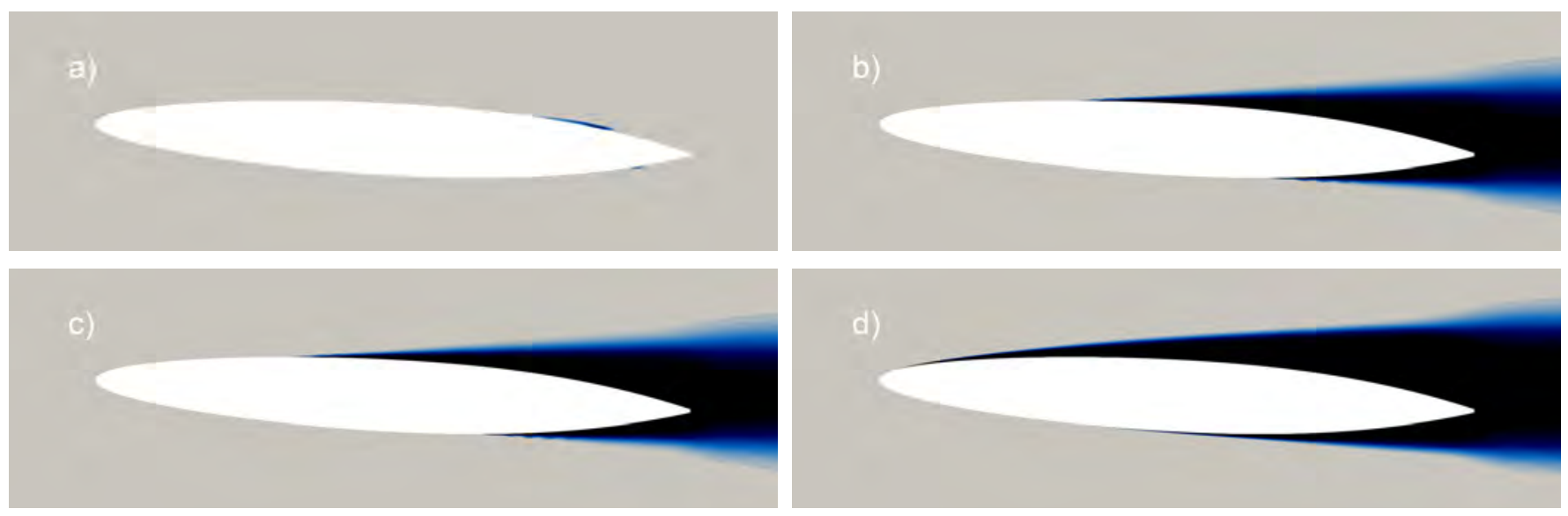

Figure 12. Sheet cavitation development history of NACA16012, $\mathrm{AoA}=3^{\circ}, \operatorname{Re}=1,000,000, \sigma=0.045$. (a-c) bridged model at $\mathrm{T}=0.001 \mathrm{~s}, 0.1 \mathrm{~s}$ and $0.3 \mathrm{~s}$. (d) unbridged $k-\omega S S T+S S$ at $\mathrm{T}=0.3 \mathrm{~s}$.
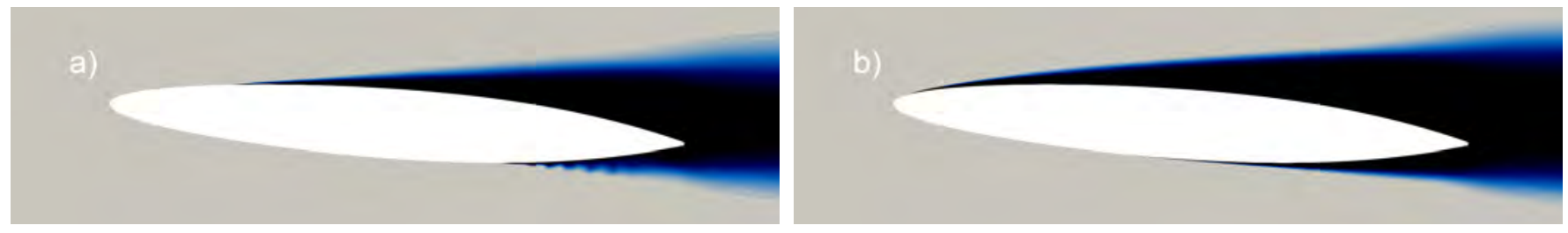

Figure 13. Sheet cavitation prediction of NACA16012, $\mathrm{AoA}=4^{\circ}, \operatorname{Re}=1,000,000, \sigma=0.045$. (a) bridged model; (b) unbridged $k-\omega S S T+S S$.
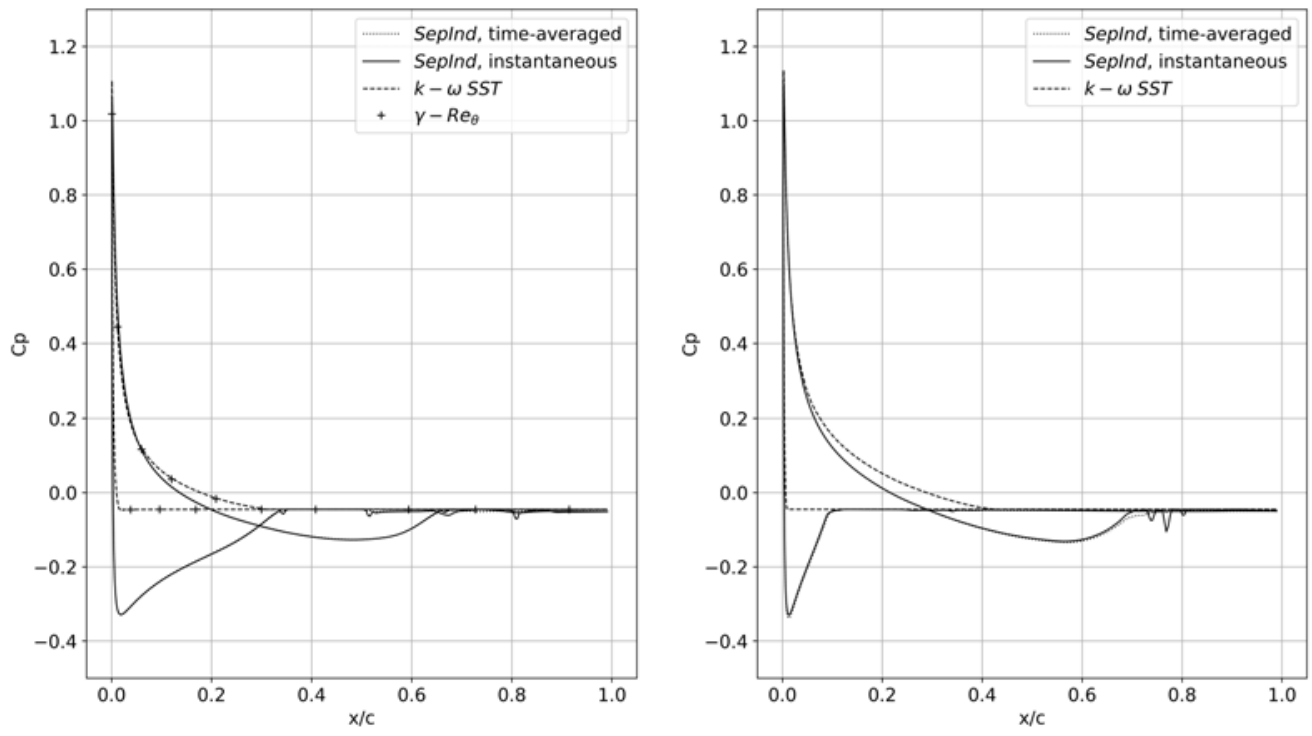

Figure 14. Predicted pressure coefficients under cavitating condition for $\mathrm{AoA}=3^{\circ}(\mathbf{l e f t})$ and $\mathrm{AoA}=5^{\circ}$ (right).

\section{The PPTC VP1304 Propeller}

\subsection{Non-Cavitating Conditions}

The predictions are shown in Figure 15 using the $\gamma-R e_{\theta}$ transition model and the $k-\omega S S T$ turbulence model in Figure 16. The SepInd is computed as in the bridged model, albeit it does not influence predictions in these non-cavitating conditions. The Q-criterion with iso-surface of $3 \times 10^{5}$ is shown colored by pressure, and the pressure legend lower limit is set to saturation pressure. Besides, the skin friction is also shown on the propeller blades with wall limiting stream lines, with predicted SepInd shown in black regions on the blade surfaces. 


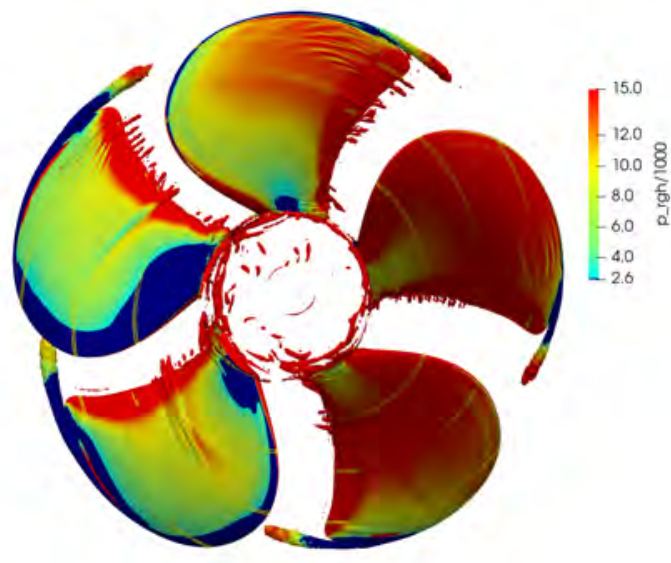

(a)

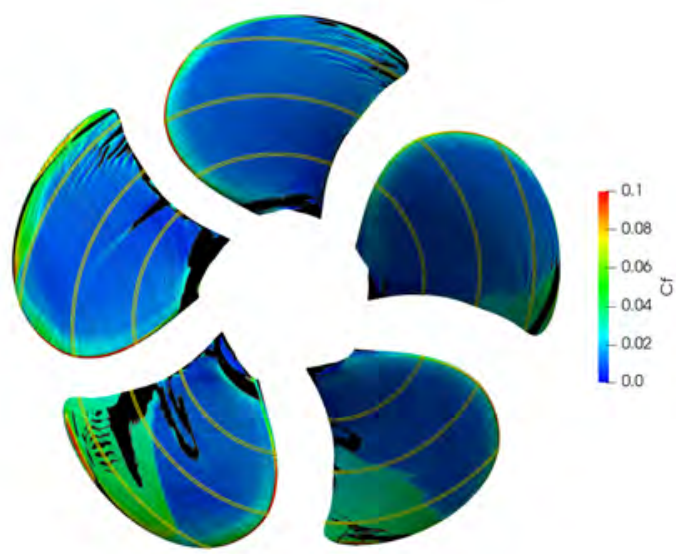

(b)

Figure 15. PPTC VP1304 mounted on $12^{\circ}$ inclined shaft, $J=1.019$, non-cavitating condition predictions using $\gamma-R e_{\theta}$ model with calculated SepInd. (a) $\mathrm{Q}=3 \times 10^{5}$ colored by pressure; (b) SepInd with wall limiting streamlines and contours of $C_{f}$.

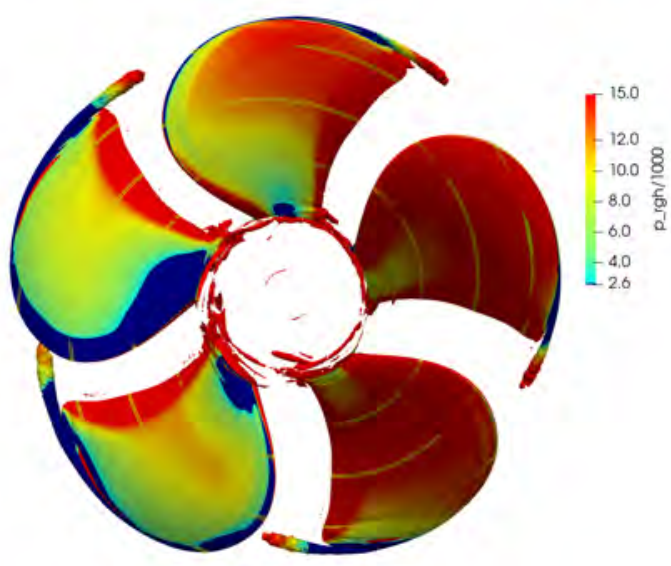

(a)

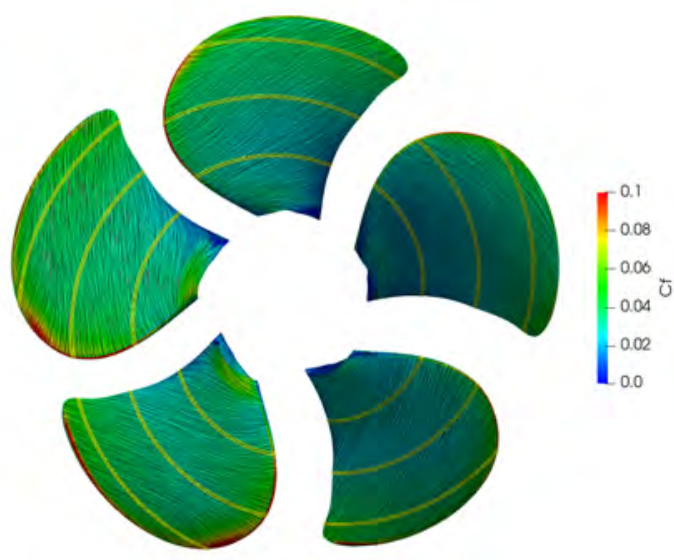

(b)

Figure 16. PPTC VP1304 mounted on $12^{\circ}$ inclined shaft, $J=1.019$, non-cavitating condition predictions using $k-\omega S S T$ model. (a) $\mathrm{Q}=3 \times 10^{5}$ colored by pressure; (b) wall limiting streamlines and contours of $C_{f}$.

The existence of laminar and transitional flow can be noticed based on the skin friction contours and the direction of wall limiting streamlines. Comparing to a turbulent boundary layer flow, regions with laminar and transitional flow have smaller values of skin friction and the wall limiting stream lines tend towards the outer radii. If define the upper-most blade position as blade position 1, and the other four blade positions to position 2, 3, 4, and 5 in the blade rotation direction (anti-clockwise in the figures), it can be found that for blade position 1 , the blade is almost covered with laminar-transitional flow, and the turbulent boundary layer region starts to increase for blade position 2, 3, 4 to finally decrease on blade position 5.

Looking at the pressure predictions, it can be found that for blade positions 1, 2, and 3 , the whole leading edge is predicted to be below saturation pressure. Further, the difference of pressure prediction between the transition sensitive model and the fully turbulent model is small. This indicates that for cavitating conditions with cavitation mass transfer determined by $p-p_{\text {sat }}$, cavitation is expected to start developing from almost the whole leading edge.

The predicted Q-criterion shows differences between using the $\gamma-\operatorname{Re}_{\theta}$ transition model and only the standard $k-\omega S S T$ turbulence model. Both models predict the leading edge vortex at the outer radii, while strips of flow structures with higher values of skin friction can be found close to the blade tip using the $\gamma-R e_{\theta}$ transition model. The blade 
leading edge vortex and the predicted strips can be related to the prediction of SepInd. An interesting observation is that on the blade positions 2 and 3, a strip of high $Q$ can be found at around $0.6 \mathrm{R}$, which also triggered the prediction of SepInd.

The predicted propulsion characteristics are summarized in Table 9. The predictions using the fully turbulent model agree well compared to experimental measurements with very close value of $K_{T}$ and under-prediction of $K_{Q}$ with about $2 \%$. Using the $\gamma-R e_{\theta}$ transition model, higher $K_{T}$ is predicted with about $4.1 \%$ compared to the experimental measurement. It can be noted that under the studied high advance ratio, transition model predictions are commonly found predicting higher $K_{T}$, possibly because of the lack of cross-flow correlations, which is not considered in the present study.

Table 9. Predicted non-cavitating propulsion characteristics.

\begin{tabular}{cccc}
\hline$J=\mathbf{1 . 0 1 9}$ & Cavitation Number $\sigma$ & $\boldsymbol{K}_{\boldsymbol{T}}$ & $\mathbf{1 0}_{\boldsymbol{Q}}$ \\
\hline $\operatorname{EXP}$ & non-cavitating & 0.392 & 1.010 \\
$\gamma-\operatorname{Re}_{\theta}$ & non-cavitating & 0.408 & 1.010 \\
$k-\omega S S T$ & non-cavitating & 0.393 & 0.990 \\
\hline
\end{tabular}

\subsection{Cavitating Conditions}

The predicted cavitation patterns using the bridged model are shown in the upper frames in Figure 17 and predictions using unbridged approach $k-\omega S S T+S S$ are shown in the lower frames. The unbridged predictions are very similar with most predictions by different institutes that participated in the SMP propeller workshop using different viscous approaches [14]. Starting from blade position 1, almost the whole leading edge is cavitating, and the sheet cavity develops massively to, at blade positions 2 and 3, cover almost the whole blade. At blade position 2, there is a small area on the leading edge at about $0.3 \mathrm{R}$ close to the blade root that is not cavitating, which is also predicted similarly with the majorities in the workshop. Comparing to the experimental observations, it is clear that the sheet cavitation is massively over-predicted, especially for blade positions 1,2 and 3, while, in the experiment, at these blade positions rather intense traveling bubble cavitation is observed. Besides, strip-like cavitation structures are quite often found developed in the experimental observations, starting from $0.7 \mathrm{R}$ close to the leading edge. Cavitation can also be found at $0.6 \mathrm{R}$ at mid-chord for blade positions 2 and 4 .

The bridged model predicts significantly improved cavitation patterns. At blade position 1, the predicted cavitation starts at around $0.95 \mathrm{R}$, which agree well with experimental observation. At blade position 2, the predicted sheet cavitation starting location is about $0.91 \mathrm{R}$, while in the experimental observation the starting location on the leading edge is located at about $0.88 \mathrm{R}$. At blade position 3, the predicted cavitation pattern agrees with experimental observation as well, but the shape of sheet cavitation closure line differs somewhat. For blade positions 4 and 5 , the predictions are almost the same as with the unbridged model predictions; both in decent agreement with experimental observations as discussed below.

The quantity SepInd are shown on the propeller blades in Figure 17 as well. It can be found that at 0.6-0.7 R at around mid-chord, a strip of non-zero SepInd is reported. In the experimental observation, strip-like cavitation can be found at blade position 2 and position 4 , and on blade position 2 this strip like cavitation is predicted by the bridged model as well at similar locations, see the blade positions of $12^{\circ}, 24^{\circ}$ and $36^{\circ}$ frames in Figure 17. This cavitation structure is also visible at $0^{\circ}$ if looking at the iso-surface of $\alpha_{v}=0.1$ (the figure shows $\alpha_{v}=0.5$ ). For blade position 4 , at $0.6 \mathrm{R}$ mid-chord, SepInd can be found predicted without cavitation showing up. Discrepancies can be found for the blade at position 4 between numerical prediction and experimental observation: in numerical simulations using both bridged and unbridged models, the sheet cavitation starts at about $0.96 \mathrm{R}$ with quite limited extents, and most numerical predictions shown in the workshop reported very similar limited cavitation pattern locally. In the experimental observations, the cavitation pattern on blade position 4 starts already at $0.92 \mathrm{R}$ and covers 
a larger region. This may suggest there are configuration differences between experimental and numerical settings.

a)

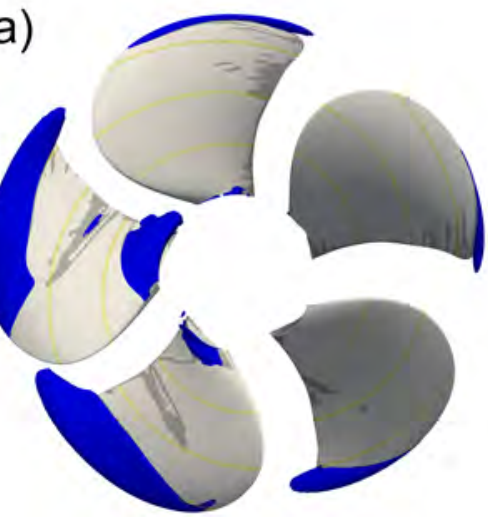

d)

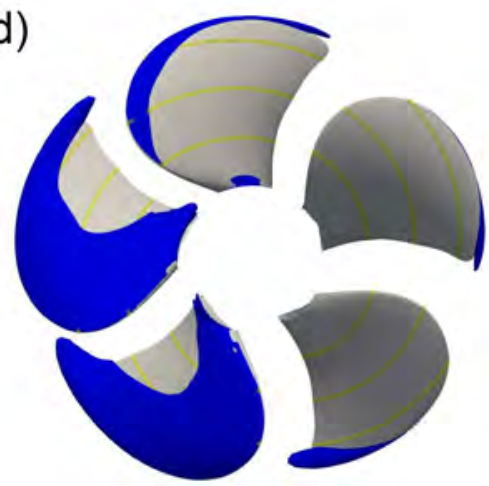

g)

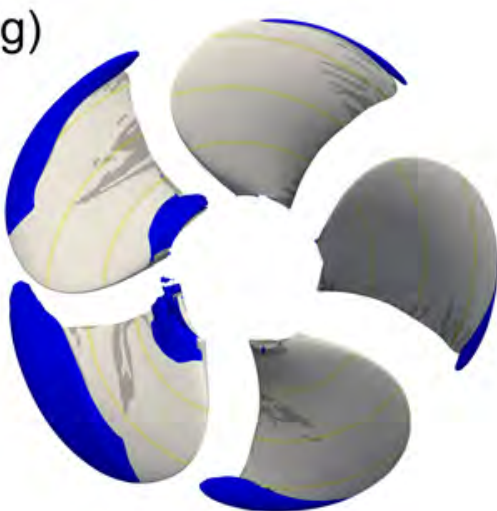

j)

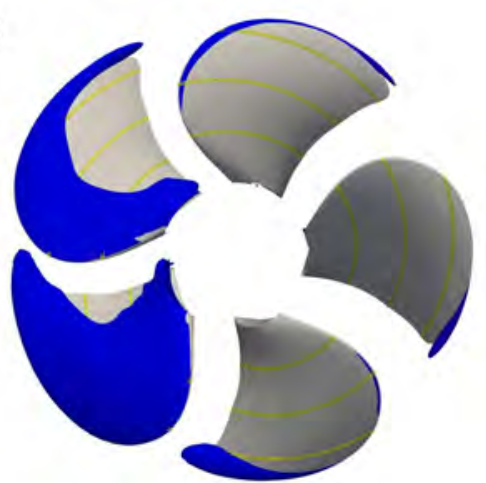

b)

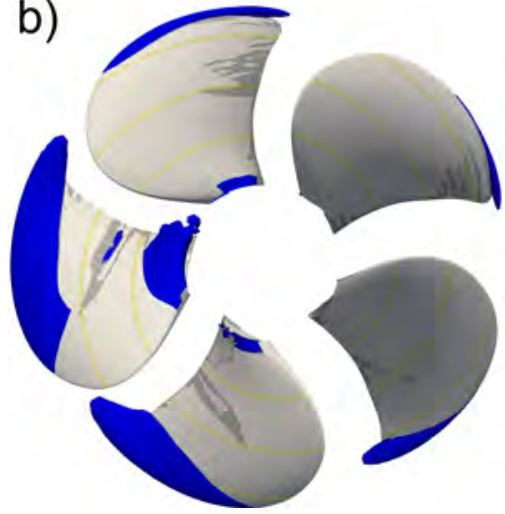

e)

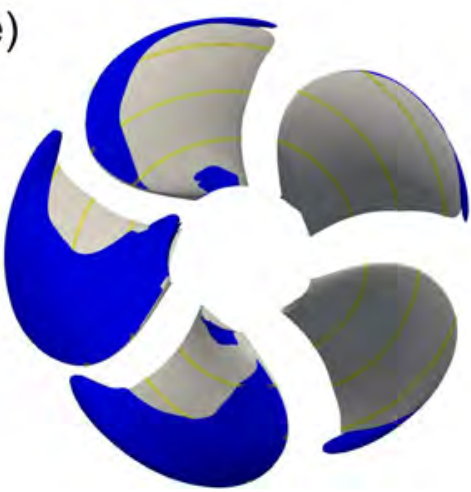

h)

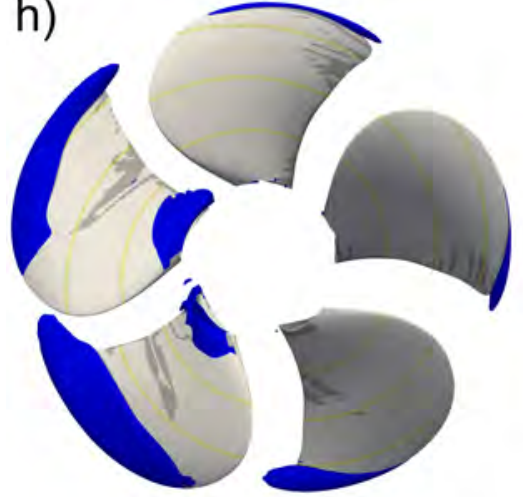

k)

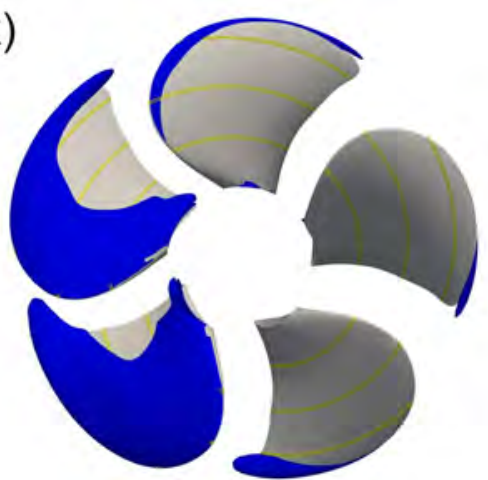

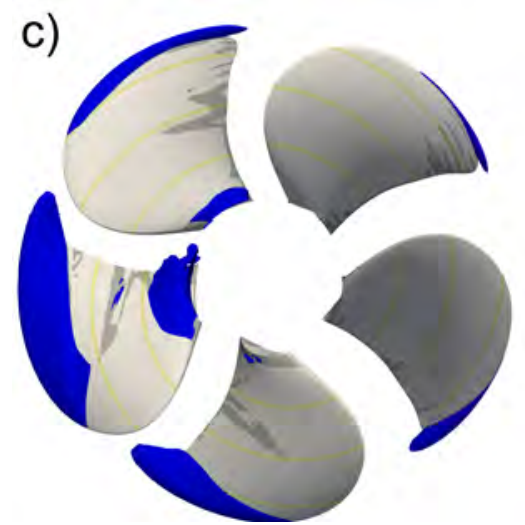

f)

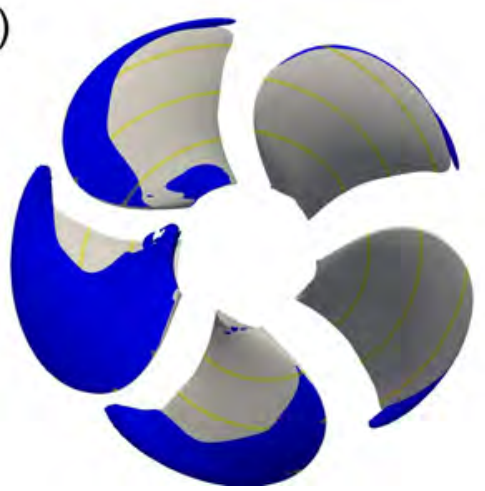

i)
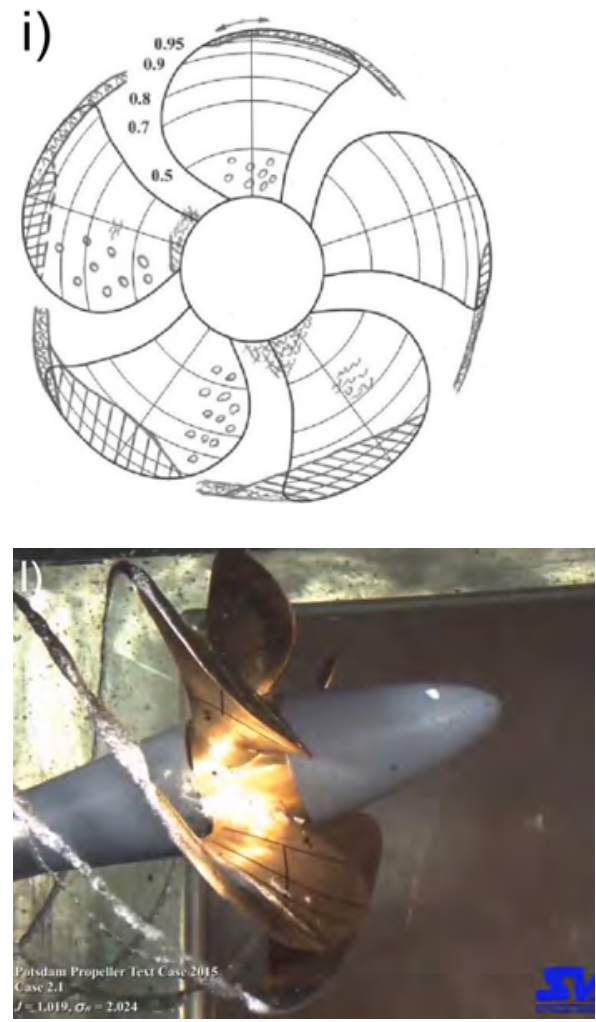

Figure 17. Predicted cavitation pattern $\left(\alpha_{v}=0.5\right)$ using the bridged model (upper frames $\left.(\mathbf{a}-\mathbf{c}, \mathbf{g}, \mathbf{h})\right)$ and $k-\omega S S T+S S$ without transition model (lower frames $(\mathbf{d}-\mathbf{f}, \mathbf{j}, \mathbf{k}, \mathbf{k})$ ) compared to the experimental sketch (i) and photo snapshot $(\mathbf{l})$. (a,d) blade phase $=12^{\circ} ;(\mathbf{b}, \mathbf{e})$ : blade phase $=24^{\circ} ;(\mathbf{c}, \mathbf{f})$ blade phase $=36^{\circ} ;(\mathbf{g}, \mathbf{j})$ blade phase $=60^{\circ} ;(\mathbf{h}, \mathbf{k})$ blade phase $=0^{\circ}$. 
The predicted propulsion characteristics $K_{T}$ and $K_{Q}$ for the cavitating conditions are shown in Table 10. The thrust breakdown, denoted $\Delta K_{T}$, is reported as well, accounting for the difference between non-cavitating and cavitating thrust coefficients. The bridged model predicted rather high $K_{T}$ compared to the experimental measurement, partly due to the inherited over-predictions under non-cavitating conditions, while if looking at $\Delta K_{T}$, the thrust breakdown is 0.016 , still significantly lower than the experimental measurement, which is 0.029 . Using the unbridged model with $k-\omega S S T+S S$, higher prediction of $K_{T}$ and lower $\Delta K_{T}$ are predicted as well; the predicted $\Delta K_{T}$ is 0.018 and smaller than the experimental thrust breakdown, but with an over-prediction of cavitation pattern. Using the bridged model, the massive sheet cavitation prediction is avoided and the low pressure region on the blade suction side remains non-cavitating, thus it is logical to predict less thrust breakdown comparing to predictions with massive over-prediction of cavitation. In the original workshop, for the participants with similar over-prediction of cavitation patterns, many under-predicted the thrust breakdown and over-predicted the cavitating $K_{T}$, while the predictions are spreading and seemingly $K_{T}$ is also sensitive to the choice of used mass transfer models. Besides, the traveling bubble cavitation on the blades in the experiments is very intense, especially on blade positions 2 and 3; this is different from the observations for the NACA16012 hydrofoil shown earlier or the following propeller case. This intense traveling bubble cavitation is suspected to be related with water quality and may also have an impact on the force predictions, but is not captured in the bridged model and predicted as sheet cavitation using the unbridged approach. Besides, there might also exist some discrepancies between numerical setting and experimental configuration, as the pre-mentioned cavitation discrepancies on blade position 4 .

Table 10. Predicted cavitating propulsion characteristics.

\begin{tabular}{ccccc}
\hline$J=\mathbf{1 . 0 1 9}$ & Cavitation Number $\sigma$ & $\boldsymbol{K}_{\boldsymbol{T}}$ & $\mathbf{1 0 K _ { Q }}$ & $\boldsymbol{\Delta} \boldsymbol{K}_{\boldsymbol{T}}$ \\
\hline EXP & 2.024 & 0.363 & 0.960 & 0.029 \\
Bridged model & 2.024 & 0.392 & 0.982 & 0.016 \\
Standard $k-\omega S S T+S S$ & 2.024 & 0.375 & 0.973 & 0.018 \\
\hline
\end{tabular}

\section{Application on Kongsberg Propeller A}

As an application, the bridged model is used to predict cavitation patterns on a commercial high-skew propeller design of Kongsberg, referred to as propeller A. The propeller was mounted on an inclined shaft and based on experimental high speed videos, similar phenomena of sheet cavitation suppression were observed as for the PPTC discussed above.

\subsection{Non-Cavitating Condition}

The $\gamma-R e_{\theta}$ transition model is sensitive to the free-stream $T u$, whose value also directly influences the model correlations, including the determination of the major parameters $\gamma_{e f f}$ and SepInd used in the present study. However, Tu are usually not reported in the experimental reports, thus non-cavitating simulations with several free-stream $T u$ were performed first. The relative differences of propulsion characteristics using $k-\omega S S T$ turbulence model and $\gamma-R e_{\theta}$ transition model with different free-stream $T u$ are summarized in Table 11 . With a very high $T u=3 \%$, the predicted $K_{T}$ and $K_{Q}$ are similar with predictions using the $k-\omega S S T$ turbulence model, while with lower $T u$ values, higher $K_{T}$ and $K_{Q}$ are predicted. This trend can be expected since with a lower $T u$ value, a larger laminar-transitional region will be predicted on the propeller blades, and with higher $\mathrm{Tu}$, a larger region with a turbulent boundary layer will be predicted and lead to the predictions similar to the standard $k-\omega S S T$ fully turbulent model. 
Table 11. Predicted relative differences of force coefficients for propeller A, $J=0.85$, noncavitating condition.

\begin{tabular}{ccccc}
\hline$J=\mathbf{0 . 8 5}$ & $\mathbf{T u}$ & rdKT & rdKQ & Turbulence Model \\
\hline & $0.1 \%$ & $2.3 \%$ & $-0.29 \%$ & $\gamma-\operatorname{Re}_{\theta}$ \\
& $0.5 \%$ & $2.5 \%$ & $0.36 \%$ & $\gamma-\operatorname{Re}_{\theta}$ \\
$1.0 \%$ & $2.0 \%$ & $0.39 \%$ & $\gamma-\operatorname{Re}_{\theta}$ \\
& $3.0 \%$ & $-2.3 \%$ & $0.21 \%$ & $\gamma-R e_{\theta}$ \\
& $0.5 \%$ & $-2.6 \%$ & $0.24 \%$ & $k-\omega S S T$ \\
\hline
\end{tabular}

In Figure 18, the predictions with $T u=0.5 \%$ using the $\gamma-R e_{\theta}$ model are shown in the upper row and predictions using $k-\omega S S T$ are shown in the lower row for comparison. The iso-surface of $Q=3 \times 10^{5}$ are shown in the left column, colored by pressure with lower legend limit set to saturation pressure, thus the region with pressure lower than saturation pressure are shown in dark blue. In the right side column, wall limiting streamlines are shown on each propeller blade that in turn are colored by skin friction $C_{f}$. In addition, the predicted SepInd are colored black predicted using the bridged model.

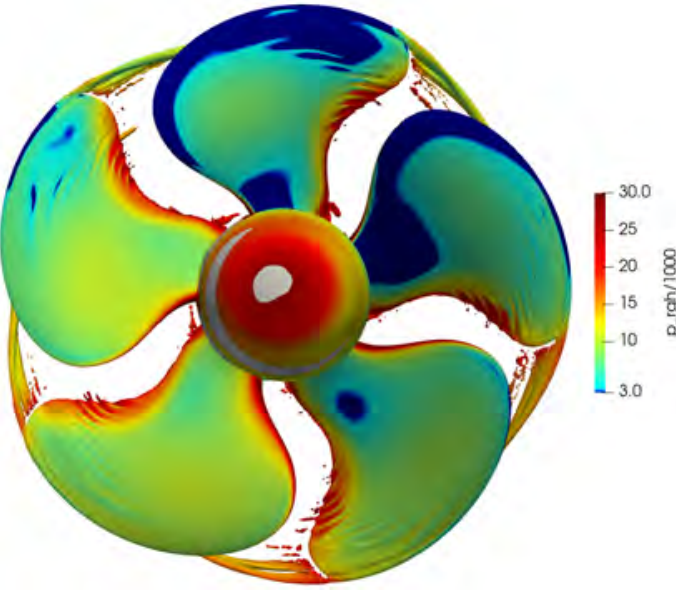

(a)

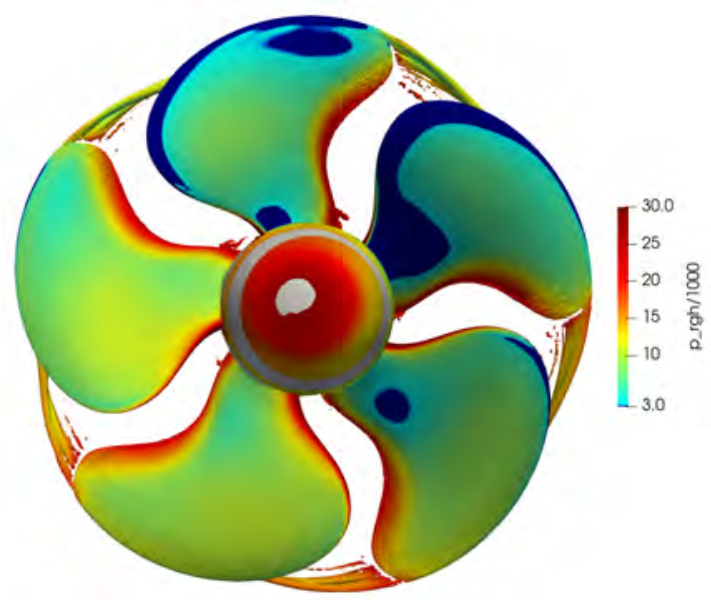

(c)

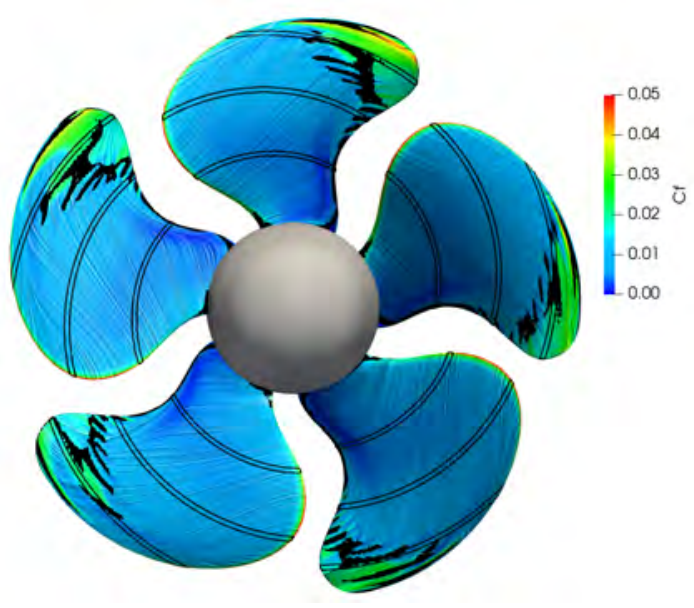

(b)

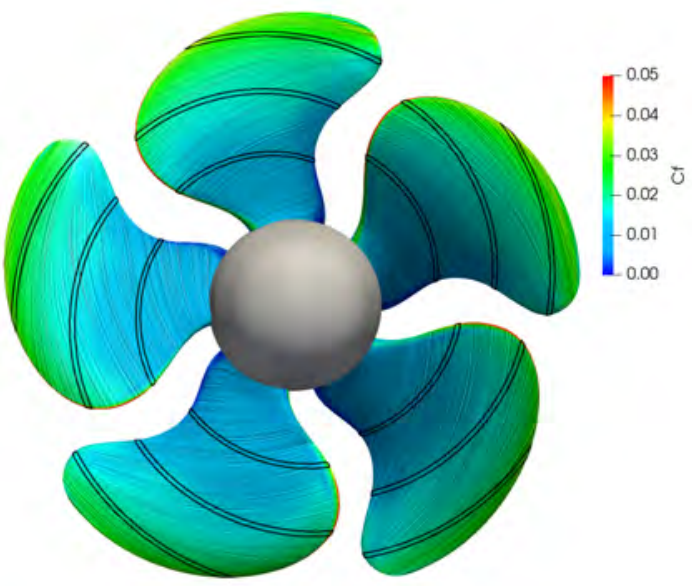

(d)

Figure 18. Non-cavitating condition predictions. $(\mathbf{a}, \mathbf{c})$ : iso-surface of $\mathrm{Q}=3 \times 10^{5}$ colored by pressure; $(\mathbf{b}, \mathbf{d})$ : predicted SepInd with wall limiting streamlines colored by $C_{f}$; with $\gamma-R e_{\theta}$ (upper row) and $k-\omega S S T$ (lower row) turbulence model.

Using the transition model, a large region of laminar-transitional flow is predicted with a set of high $Q$ strips close to the trailing edge and blade tip, leading to strip-like patterns of high skin friction. The wall-limiting streamlines are also tending outwards in radial direction compared to the predictions by $k-\omega S S T$ turbulence model, due to the relatively 
smaller skin friction in the laminar-transition region. There is no major difference in pressure prediction between the two approaches. If we number the up-straight positioned blade as blade 1 and continue in the anti-clockwise direction for the rest of the blades, for blades 1 and 5, almost the whole leading edge have pressure below saturation pressure. Consequently, if introducing a mass transfer model determined by $p-p_{\text {sat }}$ these two blades are expected to be largely covered by vapor. Studying the predicted SepInd, it can in general be found to be correlated with skin friction contours and strips of high $Q$ and develops together with several interesting flow features: at the leading edge around $0.93 \mathrm{R}$, it appears together with the leading edge vortex; at the region close to blade tip between $0.7 \mathrm{R}$ and $0.9 \mathrm{R}$, it develops on the border between the laminar-transition and turbulent regions; on the blade trailing edge, it is triggered where separation is expected due to the local blade geometry. It can be mentioned these strip-like $Q$ structures and wall shear stress can be widely found in studies using the $\gamma-R e_{\theta}$ transition model for marine propellers.

The predicted SepInd when using different Tu are shown in Figure 19, together with the skin friction. With $T u=0.1 \%$ and $T u=0.5 \%$, the predictions are rather similar with slight differences at the leading edge close to the blade tip and some strips. With increasing $T u$, larger regions of SepInd are predicted and especially with $T u=3 \%$ the blade is almost covered with turbulent boundary layer and massively predicted SepInd. With $T u=1 \%$, the whole leading edges of blades 1 and 5 are covered by SepInd. This indicates that with a free-stream $T u$ higher than $1 \%$, the predicted cavitation pattern using the bridged model can likely be similar to the predictions using unbridged models or no transition model at all.

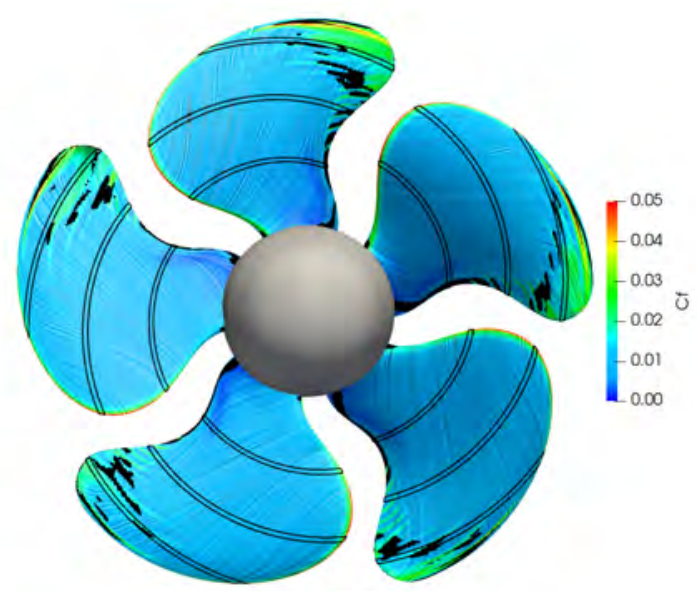

(a)

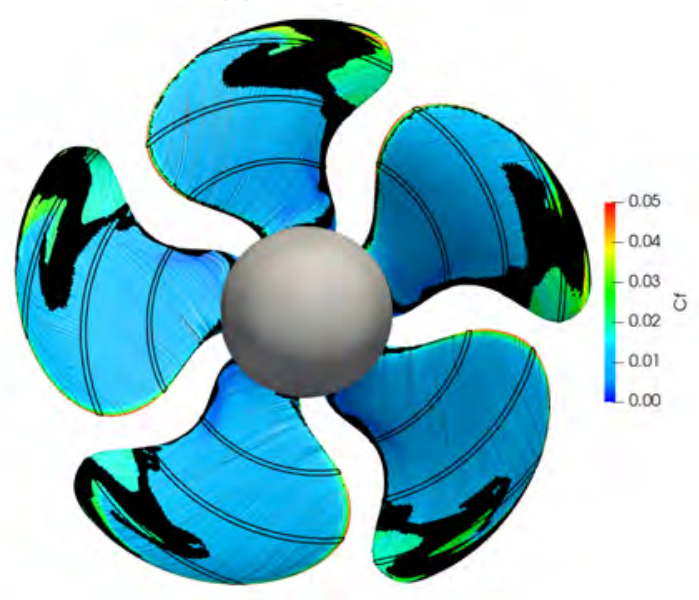

(c)

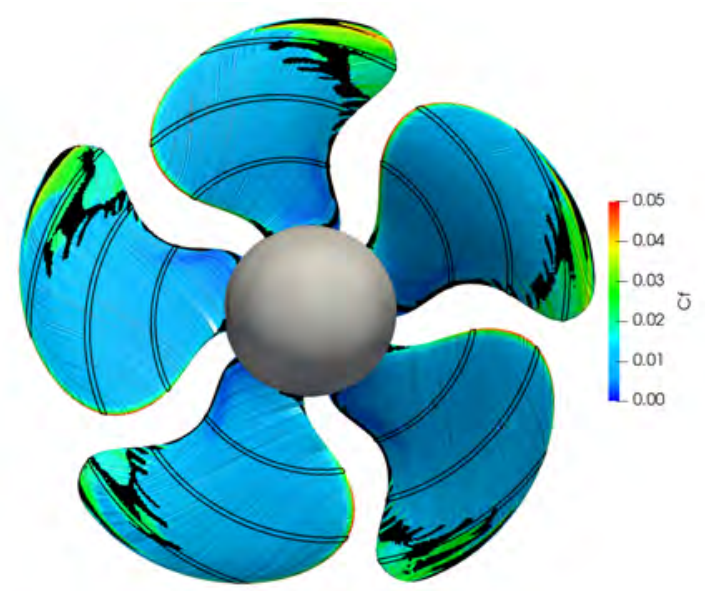

(b)

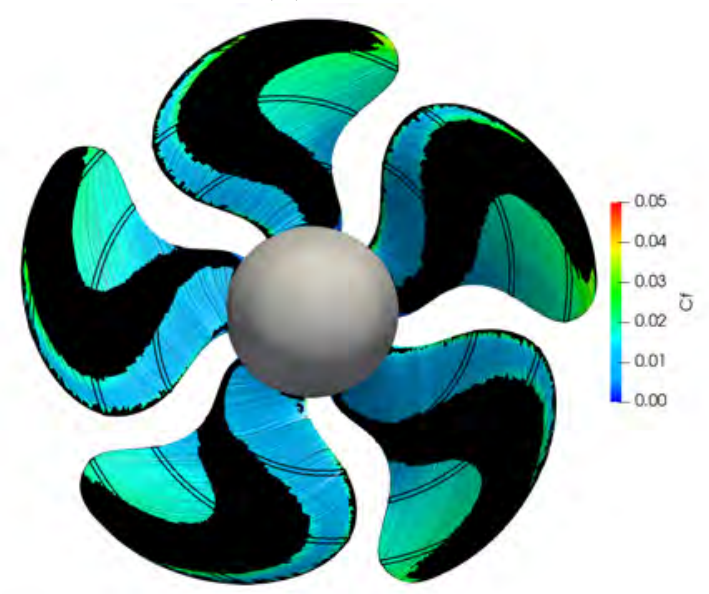

(d)

Figure 19. Predicted SepInd, wall limiting streamlines and $C_{f}$ with different $\mathrm{Tu}$ levels. (a) $\mathrm{Tu}=0.1 \%$; (b) $\mathrm{Tu}=0.5 \%$; (c) $\mathrm{Tu}=1 \%$; (d) $\mathrm{Tu}=3 \%$. 


\subsection{Cavitating Condition}

The studied operating condition here is $J=0.85$ and $\sigma=2.0$ with assumed freestream intensity level $T u=0.5 \%$. Three typical snapshots taken by the high speed video in the experiments are shown in Figure 20. The camera's shooting angle is limited, but still detailed information of cavitation formation is provided. The cavitation pattern was varying between blade revolutions, but no significant differences were found between the blades, thus the variation is not expected to be dependent on blade manufacture deficiencies nor pitch setting inaccuracies, but on water quality. Traveling bubble cavitation can be found on the propeller blades in the second and third frame, indicating that the local pressure is below saturation pressure. These traveling bubbles can not attach to the blade surface, but are transported downstream by the flow, with possible violent growth and collapse shown in the third frame as an example. The sheet cavitation typically starts developing from the leading edge at around $0.95 \mathrm{R}$, with some strip cavities starting from $0.9 \mathrm{R}$ mid-chord and develops to about $0.8 \mathrm{R}$ at the trailing edge. Tip vortex cavitation and limited root cavitation and root traveling bubble cavitation can be found for the present condition as well.
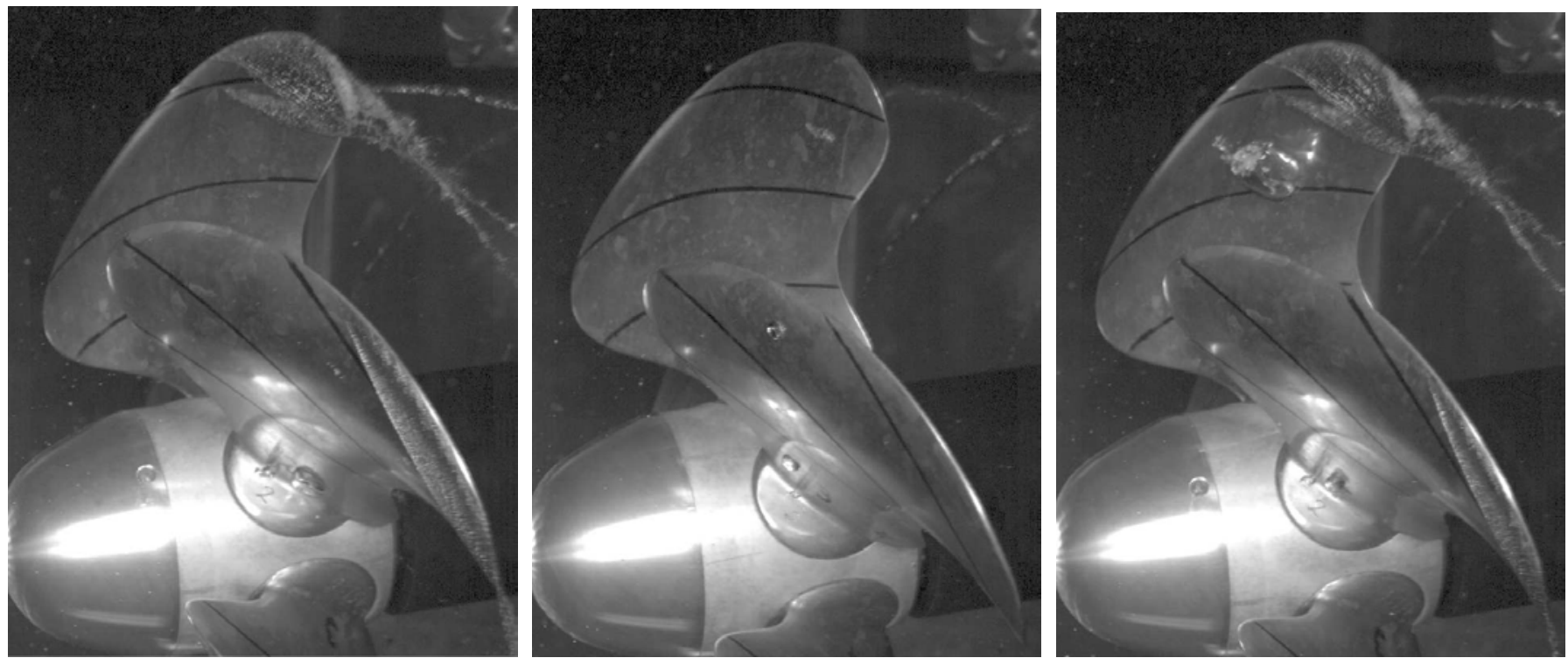

Figure 20. Typical cavitation patterns recorded in the experiments, $J=0.85, \sigma=2.0$. Left frame: typical cavitation pattern; middle frame: strip like cavitation on upper blade with traveling bubble on the lower blade; right frame: typical cavitation pattern with collapsing bubble.

The predicted cavitation patterns using the $k-\omega S S T+S S$ unbridged model is shown in the left frame in Figure 21. It can be seen clearly that the sheet cavitation is massively over-predicted, especially on blade 5 more than half of the blade is covered with sheet cavitation. The predictions using the bridged model agrees very well with the observations with sheet cavitation starting at about $0.95 \mathrm{R}$ on the leading edge. On the upper blade 1 , the overall cavitation pattern also agrees with experimental observation, and it can be found that the cavitation consists of two major structures: one generated from the leading edge $(0.95 \mathrm{R})$ and transported downstream; the other one originating from strip-like cavitation at around $0.85 \mathrm{R}$ at mid-chord, while the shape of the latter varies a bit. On the lower blade 4 , strip-like cavitation is also predicted, but in the experimental videos, these structures are very intermittently observed. As noted above, such strip-like cavitation structures are not rare for model scale marine propellers. One further example is the high speed video snapshots in [25], where series of strip-like cavitation structures close to the blade tip are formed. 

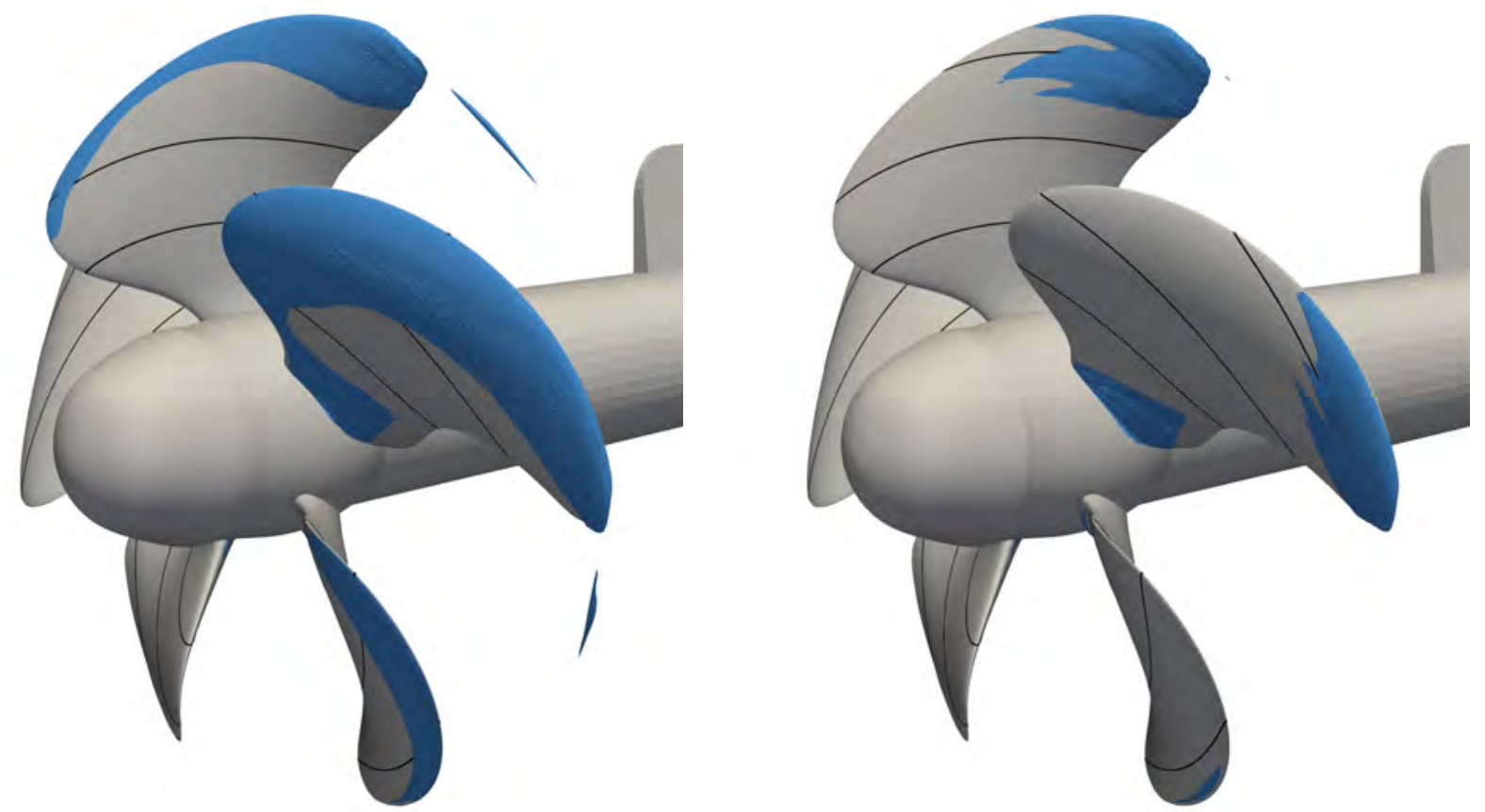

Figure 21. Predicted cavitation patterns $\alpha_{v}=0.5, J=0.85, \sigma=2.0$. Left frame: unbridged $k-\omega S S T+S S$; right frame: bridged model.

It can be noticed that the tip vortex cavitation is predicted using the unbridged model, but in the present model, the $\gamma-R e_{\theta}$ and the corresponding bridging is only effective inside the boundary layer without predicting tip vortex cavitation. Further development is needed to make the laminar cavitation suppression active only in the boundary layer and allow for cavitation to appear elsewhere.

\section{Summary}

In the present study, the local correlation based transition model $\gamma-R e_{\theta}$ is bridged with the Schnerr-Sauer cavitation mass transfer model via the laminar separation correlation in the former. This bridging is used to modify the vaporization rate thereby suppressing cavitation inception in laminar boundary layers. Apart from adjusting the vaporization constant, the transition and mass transfer models are kept intact.

The bridged model is first developed and applied on the NACA16012 hydrofoil with different angles of attack and predictions are compared to published experimental data. Very satisfying predictions of cavitation pattern are achieved for several tested angles of attack while the unbridged approach predicts rather massive sheet cavitation. The prediction of laminar separation location agreed well with experimental data with reported free-stream turbulence intensity level $\mathrm{Tu}$ for non-cavitating conditions.

Then, the bridged model is used for predicting the cavitation patterns on model scale propellers mounted on inclined shafts. The first propeller test case is the PPTC VP1304 model scale marine propeller, used in the second international workshop on cavitation and propeller performance [14]. The used test condition is referred to as case 2.1 in the workshop. Under this condition, most workshop participants reported massive sheet cavitation predictions where the experimental observations showed bubble cavitation. Using the unbridged approach, similar over-prediction of sheet cavitation was found covering large part of the propeller blades. The bridged model gave predictions of sheet cavitation pattern, including cavitation strips at blade mid-chord and mid-radius, as observed in the experiments. The second studied case is a commercial high-skew propeller design of Kongsberg. For non-cavitating conditions, simulations with varying $\mathrm{Tu}$ were performed and the influences on propeller blades was studied. For the cavitation conditions, the predictions are compared to experimental high speed video snapshots. The cavitation pat- 
terns predicted using the bridged model agreed very well with experimental observations, significantly improved compared to the unbridged approach that again predicted massive sheet cavitation on the blades.

Regarding the bridging, it relies on the local correlations in the original $\gamma-\operatorname{Re}_{\theta}$ transition model which triggers the separation induced transition. The correlation in essence depends on the free-stream turbulence level $T u$, local pressure gradient, and local vorticity Reynolds number $R e_{v}$ and is related to the local boundary layer shape factor $H$. The predicted laminar separation locations have been reported to be satisfying in the literature. The use of this parameter for sheet cavitation inception prediction is inline with existing knowledge regarding the mechanisms of sheet cavitation inception. This includes experimental observations that laminar separation is a prerequisite for sheet cavitation inception, or the more recent findings suggesting the existence of adverse pressure gradient and local thickening of boundary layer is sufficient. However, it is also necessary to remark that the bridging thus relies on the prescribed local correlations, which have a physical meanings, but are not fully physically formulated. Besides, the transition correlations are based on single fluid experimental series and the knowledge about multiphase turbulent and transitional flow is limited. Still, the prediction results using the bridged model are very satisfying, awaiting advanced approaches.

Author Contributions: Conceptualization, methodology, formal analysis, investigation, writing —original draft preparation, M.G.; resources, U.S. and R.E.B.; writing-review and editing, U.S. and R.E.B.; supervision, U.S. and R.E.B.; project administration, R.E.B.; funding acquisition, R.E.B. All authors have read and agreed to the published version of the manuscript.

Funding: This research was funded by Konsberg Maritime Sweden AB through the University Technology Centre in Computational Hydrodynamics hosted at the Department of Mechanics and Maritime Sciences at Chalmers University of Technology.

Institutional Review Board Statement: Not applicable.

Informed Consent Statement: Not applicable.

Data Availability Statement: Data is contained within the article.

Acknowledgments: The computations were performed on resources at Chalmers Centre for Computational Science and Engineering (C3SE) provided by the Swedish National Infrastructure for Computing (SNIC).

Conflicts of Interest: The authors declare no conflict of interest.

\section{References}

1. Franc, J.P.; Michel, J.M. Fundamentals of Cavitation; Springer Science \& Business Media: Dordrecht, The Netherlands, 2006; Volume 76.

2. Franc, J.P.; Michel, J.M. Attached cavitation and the boundary layer: Experimental investigation and numerical treatment. J. Fluid Mech. 1985, 154, 63-90. [CrossRef]

3. Franc, J.P.; Michel, J.M. Unsteady attached cavitation on an oscillating hydrofoil. J. Fluid Mech. 1988, 193, 171-189. [CrossRef]

4. Casey, M. The inception of attached cavitation from laminar separation bubbles on hydrofoils. In Proceedings of the IME Conference on Cavitation at Heriot-Watt University, Edinburgh, UK, 3-5 September 1974; Volume 9.

5. Arakeri, V.H. Viscous effects on the position of cavitation separation from smooth bodies. J. Fluid Mech. 1975, 68, 779-799. [CrossRef]

6. Croci, K.; Ravelet, F.; Danlos, A.; Robinet, J.C.; Barast, L. Attached cavitation in laminar separations within a transition to unsteadiness. Phys. Fluids 2019, 31, 063605. [CrossRef]

7. Van der Meulen, J.H. Boundary layer and cavitation studies of NACA 16-012 and NACA 4412 hydrofoils. In Proceedings of the 13th Symposium on Naval Hydrodynamics, Tokyo, Japan, 6-10 October 1980; pp. 195-219.

8. Arakeri, V.; Acosta, A. Viscous effects in the inception of cavitation on axisymmetric bodies. J. Fluids Eng. 1973, 95, 519-527. [CrossRef]

9. Arndt, R.E. Cavitation in fluid machinery and hydraulic structures. Annu. Rev. Fluid Mech. 1981, 13, 273-326. [CrossRef]

10. Ducoin, A.; Astolfi, J. Wall-pressure fluctuations of laminar separation bubble based on direct numerical simulation and experiments over a hydrofoil at $\operatorname{Re}=450,000$. Eur. J. Mech.-B/Fluids 2019, 76, 132-144. [CrossRef] 
11. Gopalan, S.; Katz, J. Flow structure and modeling issues in the closure region of attached cavitation. Phys. Fluids 2000, 12, 895-911. [CrossRef]

12. Ram, O.; Agarwal, K.; Katz, J. On the mechanisms that sustain the inception of attached cavitation. J. Fluid Mech. 2020, 901. [CrossRef]

13. Kuiper, G. Cavitation Inception on Ship Propeller Models. Ph.D. Thesis, Delft University of Technology, Delft, The Netherlands, 1981

14. Second International Workshop on Cavitation and Propeller Performance; Technical Report; SVA (Schiffbau-Versuchsanstalt Potsdam): Potsdam, Germany, 2015.

15. Ye, C.; Wang, F.; Wang, C.; van Esch, B.P. Assessment of turbulence models for the boundary layer transition flow simulation around a hydrofoil. Ocean Eng. 2020, 217, 108124. [CrossRef]

16. Menter, F.R.; Langtry, R.B.; Likki, S.; Suzen, Y.; Huang, P.; Völker, S. A correlation-based transition model using local variables-Part I: Model formulation. J. Turbomach. 2006, 128, 413-422. [CrossRef]

17. Langtry, R.B.; Menter, F.R. Correlation-based transition modeling for unstructured parallelized computational fluid dynamics codes. AIAA J. 2009, 47, 2894-2906. [CrossRef]

18. Langtry, R.B. A Correlation-Based Transition Model Using Local Variables for Unstructured Parallelized CFD Codes. Ph.D. Thesis, Universität Stuttgart, Stuttgart, Germany, 31 May 2006.

19. Reverberi, A.; Lloyd, T.; Vaz, G. Towards cavitation modelling accounting for transition effects. In Proceedings of the 219th Numerical Towing Tank Symposium (NuTTS), St. Pierre d'Oleron, France, 3-4 October 2016.

20. Schnerr, G.H.; Sauer, J. Physical and numerical modeling of unsteady cavitation dynamics. In Proceedings of the Fourth International Conference on Multiphase Flow, ICMF, New Orleans, LA, USA, 27 May-1 June 2001; Volume 1.

21. Menter, F.; Esch, T. Elements of industrial heat transfer predictions. In Proceedings of the 16th Brazilian Congress of Mechanical Engineering (COBEM), Uberlândia, Brazil, 26-30 November 2001; Volume 109, p. 650.

22. Menter, F.R.; Kuntz, M.; Langtry, R. Ten years of industrial experience with the SST turbulence model. Turbul. Heat Mass Transf. 2003, 4, 625-632.

23. Ge, M.; Svennberg, U.; Bensow, R.E. Numerical investigation of pressure pulse prediction for propellers mounted on an inclined shaft. In Proceedings of the Sixth International Symposium on Marine Propulsors, Rome, Italy, 26-30 May 2019.

24. Gaggero, S. Influence of laminar-to-Turbulent transition on model scale propeller performances. Part II: Cavitating conditions. Ships Offshore Struct. 2020, 1, 1-20. doi.org/10.1080/17445302.2020.1866819 [CrossRef]

25. Lu, N.X.; Bensow, R.E.; Bark, G. Large eddy simulation of cavitation development on highly skewed propellers. J. Mar. Sci. Technol. 2014, 19, 197-214. [CrossRef] 